Solid Earth Discuss., https://doi.org/10.5194/se-2017-75

Manuscript under review for journal Solid Earth

Discussion started: 6 November 2017

(c) Author(s) 2017. CC BY 4.0 License.

1

2

3

4

5

6

7

8

9

\title{
Phytoextraction and the economic perspective of phytomining of heavy metals
}

Amjad Ali ${ }^{\mathrm{a}}$, Di Guo ${ }^{\mathrm{a}}$, Amanullah Mahar, ${ }^{\mathrm{a}, \mathrm{b}}$, Wang Ping ${ }^{\mathrm{a}}$, Fazli Wahid ${ }^{\mathrm{c}}$, Feng Shen ${ }^{\mathrm{a}}$, Ronghua $\mathrm{Li}^{\mathrm{a}}$, Zengqiang Zhang ${ }^{\mathrm{a}, *}$

${ }^{\text {a }}$ College of Natural Resources and Environment, Northwest A\&F University, Yangling, 712100, China

${ }^{\mathrm{b}}$ Centre for Environmental Sciences, University of Sindh, Jamshoro, 76080, Pakistan

${ }^{c}$ Department of Soil and Environmental Sciences, The University of Agriculture, Peshawar, 25130, Pakistan

* Corresponding author: College of Natural Resources and Environment, Northwest A\&F University, Yangling, Shaanxi, 712100, China

E-mail address: zhangzq58@126.com, zqzhang@nwafu.edu.cn

Abstract: The world rapid growing population, expanding economics and anthropogenic activities contribute to heavy metals pollution, which are non-biodegradable, persistent and threaten the environment. The rising level of heavy metals in environment emphasizes on indigenous technologies, but conventional technologies are too expensive, laborious and result in secondary pollution. Phytoremediation/phytoextraction is a plant based technology, which is environmental friendly, economic and effective for heavy metals remediation. The global market of phytoremediation is $34-54$ billion US\$ and is expanding in the developed countries, providing an opportunity for this green technology. Suitability of phytoextraction depends on biomass production, accumulation rate and tolerance to target metals. Metals 
Solid Earth Discuss., https://doi.org/10.5194/se-2017-75

Manuscript under review for journal Solid Earth

Discussion started: 6 November 2017

(c) Author(s) 2017. CC BY 4.0 License.

1 uptake can be enhanced by exploring effective hyperaccumulators, expanding phytomining

2 operations and extending molecular studies on accumulation mechanism, tolerance and

3 sensitivity of heavy metals. Hyperaccumulator plants achieve greater performance at low

4 cost than conventional technologies for in situ metal removal. Phytomining generate

5 revenue and provide new research area for biofortification of food and feed, biofuel and

6 metal rich biochar production in future. This review highlights the sources of heavy metals

7 and its effects on plants, enhancing phytoremediation process and increasing economic

8 benefits of phytomining.

9 Keywords: Cadmium; Environment; Hyperaccumulator; Phytoextraction; Phytomining.

3. Phytoremediation of heavy metals: An environment friendly green technology

4. Phytoextraction (phytoaccumulation) of $\mathrm{Cd}$ and $\mathrm{Pb}$

5. Induced and natural phytoextraction of $\mathrm{Cd}$ and $\mathrm{Pb}$.....

8. Factors affecting phytoextract on (phytomining) 
Solid Earth Discuss., https://doi.org/10.5194/se-2017-75

Manuscript under review for journal Solid Earth

Discussion started: 6 November 2017

(c) Author(s) 2017. CC BY 4.0 License.

1

2

4

5

\section{Introduction}

Anthropogenic and geogenic activities contribute to heavy metals (HMs) pollution in air, soil and water bodies. Heavy metals having higher densities $\left(>5 \mathrm{gcm}^{-3}\right)$, include cadmium $(\mathrm{Cd})$, lead $(\mathrm{Pb})$, mercury $(\mathrm{Hg})$, zinc $(\mathrm{Zn})$, chromium $(\mathrm{Cr})$ and arsenic (As) etc, generally refers to metals and metalloids (Li et al., 2014). Heavy metals are considered as toxic, nonbiodegradable and extremely persistent elements in the soil and environment (Bharti and Kumar Banerjee, 2012; Luo et al., 2005; Zhao et al., 2010; Zhou et al., 2014). Heavy metals pollution is a worldwide concern, and the number of contaminated sites increasing with the passage of time due to burgeoning populations, disarrayed industrialization and expanding economics (Kaimi et al., 2006).

Industrialization improved the living standard of man, meanwhile posed numerous health and environmental threats. Global industrialization and technological innovations over the past two centuries has resulted in widespread contamination of the environment. Every factory discharge effluents, mostly containing various contaminants like $\mathrm{Cd}, \mathrm{Pb}, \mathrm{Hg}$, $\mathrm{As}, \mathrm{Zn}, \mathrm{As}, \mathrm{Cu}, \mathrm{Ni}, \mathrm{Co}, \mathrm{Se}$, and $\mathrm{Zn}$ into soil and water resources like sea, rivers and canals (Arias-Estévez et al., 2008; Daud et al., 2013). These contaminants cause catastrophic effects on human, animals and environment due to soil-plant transportation of HMs (Meighan et al., 2011; Vollenweider et al., 2006; Xiong, 1997). Naturally HMs are introduced through the weathering of parent materials, wind-blown dust (erosion), forest fires and atmospheric emissions from volcanic eruptions. Sedimentary rocks (black shale) are considered as the main sources of $\mathrm{Cd}$ and $\mathrm{Pb}$ containing $0.1-11$ and $1-150 \mu \mathrm{g} \mathrm{g}^{-1}$, 
Solid Earth Discuss., https://doi.org/10.5194/se-2017-75

Manuscript under review for journal Solid Earth

Discussion started: 6 November 2017

(c) Author(s) 2017. CC BY 4.0 License.

respectively. The natural earth crust content of $\mathrm{Cd}$ and $\mathrm{Pb}$ is ranged between $0.15-0.20$ and 10-20 $\mu \mathrm{g} \mathrm{g}^{-1}$, respectively (Arain et al., 2008; Bu et al., 2016).

The conventional methods for remediation of soil heavy metals are ineffective due to high cost, require special treatment plants and release secondary pollutants into the environment. Phytoremediation is cheap and efficient method used for in situ site remediation. Phytoremediation permanently remove the bioavailable fraction of contaminants, minimal site disturbance and is well-suited with risk-based contaminated land management systems (Jiang et al., 2015). The phytoremediation market is assumed to be 34-54 billion US\$ and is further expanding with the industrial race among the nations. Number of plants species, like Sedum alfredii, Thlaspi caerulescens, Helianthus annuus, Brassica juncea and Salix are known to extract $\mathrm{Cd}$ and $\mathrm{Pb}$ from soil-water system (Escarre et al., 2000; Lomonte et al., 2010; Meighan et al., 2011; Sun et al., 2009b; Zaier et al., 2010b). These hyperaccumulators have the potential to achieve greater performance at low cost than conventional technologies for metals removal (Bolan et al., 2014; Salazar and Pignata, 2013).

The remediation of heavy metals polluted sites through phytoextraction (phytomining) is cheaper and more effective as compared to chemical treatments (Ha et al., 2011; Li et al., 2014). Biofortification of food products, production of biofuel as new energy resource, acquiring reclaimed land for agriculture and commercial purpose and biochar for climate change mitigation, provides a new insight into the phytoremediation of HMs. Phytoremediation indirectly increase soil carbon content, retain nutrients and improve soil biochemical processes (Sheoran et al., 2013). This review gives an overview of the source 
Solid Earth Discuss., https://doi.org/10.5194/se-2017-75

Manuscript under review for journal Solid Earth

Discussion started: 6 November 2017

(c) Author(s) 2017. CC BY 4.0 License.

1 and potential effects of heavy metals, possibility of enhancing the phytoremediation

2 technology as well as an insight into the economic perspective of reclaiming contaminated

3 sites.

\section{2. Sources of heavy metals and their effects on plants}

5 Anthropogenic sources of HMs include textile, pesticides, petrochemical, energy and

6 power, leather, construction, steel manufacturing, food processing waste disposal, waste

7 incineration, mining and smelting, military operations as well as coal combustion

8 (Bhargava et al., 2012; Mahar et al., 2016; Zhao et al., 2010). A number of natural and

9 anthropogenic activities contributed to $\mathrm{Cd}$ and $\mathrm{Pb}$ contamination in the environment are presented in Fig. 1.

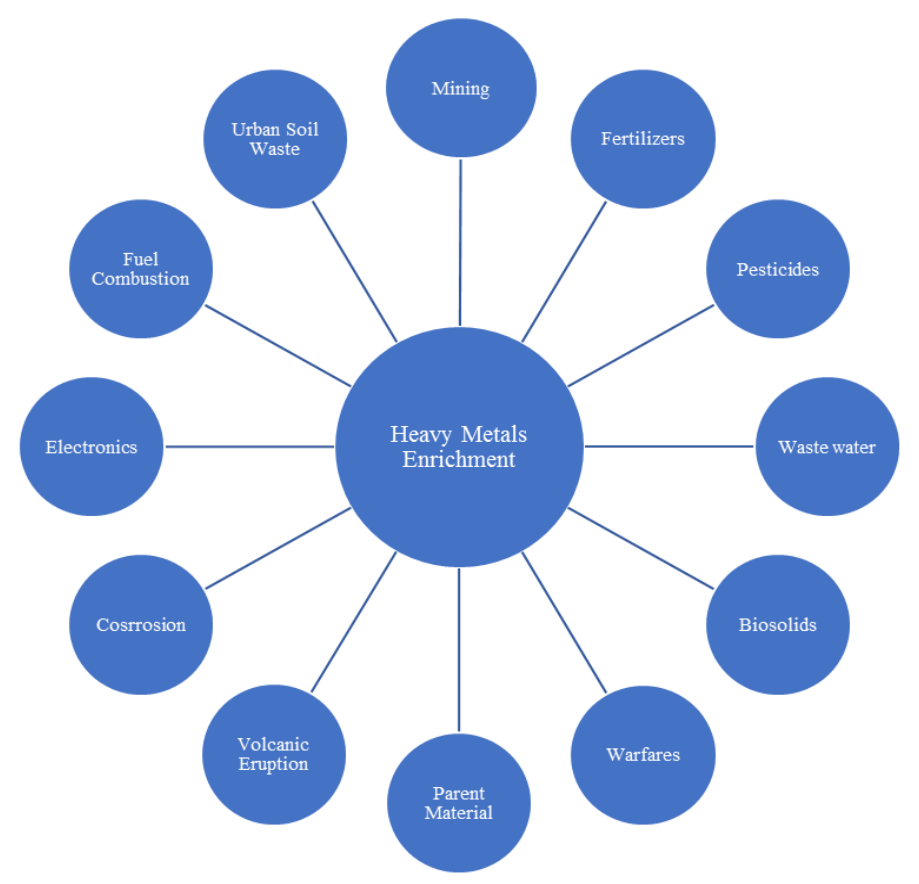

Figure 1. Natural and anthropogenic sources of heavy metals 
Solid Earth Discuss., https://doi.org/10.5194/se-2017-75

Manuscript under review for journal Solid Earth

Discussion started: 6 November 2017

(c) Author(s) 2017. CC BY 4.0 License.

1

2

4

5

\subsection{Cadmium $(C d)$}

Naturally soil $\mathrm{Cd}$ content is reported to be $0.1-0.5 \mathrm{mg} \mathrm{kg}^{-1}$, but literature has also reported the highest content up to $150 \mathrm{mg} \mathrm{kg}^{-1}$ in sites near batteries, plastics and paint manufacturing, mining, electroplating, alloy preparation, fertilizers, fungicides/pesticide, rubber tires industries, sludge and composting facilities (Gallego et al., 2012). Cadmium is considered as persistent, inorganic and toxic metal for human and plant at a low concentration (Wahid et al., 2008). Among the heavy metals, cadmium is highly soluble, causes soil pollution and adverse effects on plant growth and development. Cadmium can be taken up by plants as $\mathrm{Cd}^{+2}$ from soil solution and enter the food web. If plants exposed to high levels of $\mathrm{Cd}^{+2}$, it can affect water and elemental transportation, absorption, oxidative phosphorylation in mitochondria, photosynthesis, reduce mitochondrial respiration, growth and reproduction of plant (Padmaja et al., 1990). Cd can reduce root growth, cause cell death and chlorosis as well as inhibit auxin homeostasis and enzyme activities (Daud et al., 2013).

\section{2. $\operatorname{Lead}(\mathrm{Pb})$}

The world rapid social and economic development increased the $\mathrm{Pb}$ concentration in urban and industrial areas (Dermont et al., 2008). In 1923, lead in the form of tetraethyl lead $\left[\left(\mathrm{CH}_{3} \mathrm{CH}_{2}\right)_{4} \mathrm{~Pb}\right]$ was introduced as an anti-knock agent in fuel, which increased the lead concentration in the atmosphere (Walraven et al., 2014). Lead is released from automobile exhaust (tetraethyl lead), mining and smelters, fertilizers, pesticides, pigments, batteries, ammunition, cable sheathing, fossil fuels, manure, sludge, electricity and heat production.

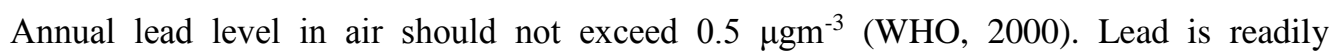


Solid Earth Discuss., https://doi.org/10.5194/se-2017-75

Manuscript under review for journal Solid Earth

Discussion started: 6 November 2017

(c) Author(s) 2017. CC BY 4.0 License.

1 adsorbed in soil, contributes to atmospheric deposition, released by natural weathering

2 processes and considered as a notorious environmental pollutant (Nagajyoti et al., 2010).

3 Lead level in ambient air ranges from $7.6 \times 10^{5}$ to $>10 \mu \mathrm{gm}^{-3}$ in remote areas (Antarctica)

4 and stationary sources (smelters), respectively (ATSDR, 2007). The lead concentration even

5 up to $300 \mathrm{mg} \mathrm{kg}^{-1}$ is also reported in roadside soils (Chen et al., 2010). The legislations in

6 1970s in Europe against the use of lead in petrol, helped in reducing the $\mathrm{Pb}$ level within a

7 safe limit (Pacyna et al., 2009). Chemical forms of lead depend on the source. Like in

8 atmosphere lead exists in the form of $\mathrm{PbSO}_{4}$ and $\mathrm{PbCO}_{3}$, coal combustion release $\mathrm{PbCl}_{2}$,

$9 \mathrm{PbO}, \mathrm{PbS}$ and insoluble mineral particles, and oil combustion mainly in the $\mathrm{PbO}$ form

10 (Wadge and Hutton, 1987). Lead particle size ranges between 0.1 and $1.0 \mu \mathrm{m}$ depending on

11 the source of emission. Lead particles in atmosphere are deposited in the terrestrial and

12 aquatic ecosystem by dry or wet deposition (Pan and Wang, 2014). Pb toxicity includes the

rapid cessation of root, stunted plant growth and chlorosis. Lead inhibits the activity of

enzymes due to its high affinity for sulphydryl groups, disturbs mineral nutrition, water

balance and alters plant hormonal status (Gopal and Rizvi, 2008). Pb increases metal containing antioxidant enzyme i.e. superoxide dismutase (SOD).

Due to such adverse effects on plants, the concern over the safe remediation invasive and aesthetically pleasing. 
Solid Earth Discuss., https://doi.org/10.5194/se-2017-75

Manuscript under review for journal Solid Earth

Discussion started: 6 November 2017

(c) Author(s) 2017. CC BY 4.0 License.

\section{3. Phytoremediation of heavy metals: An environment friendly green technology}

2 Heavy metals pollution has become a global environmental threat, which is caused by a

3 number of metals such as cadmium, lead, copper and mercury etc. (Xu et al., 2012). Some

4 plants species reported in literature, exhibit tolerance to $\mathrm{HMs}$ especially $\mathrm{Cd}$ and $\mathrm{Pb}$ (Chen et

5 al., 2014; Lomonte et al., 2010; Mahar et al., 2016; Salazar and Pignata, 2013). The rise in

$6 \mathrm{Cd}$ and $\mathrm{Pb}$ content in environment, caused by anthropogenic activities, stress the need for a

7 sustainable indigenous remediation technology. Different remediation techniques are

8 practiced for HMs polluted soils as shown in Fig. 2. But most of them are expensive,

9 laborious and cause secondary pollution as well as soil disturbance, thus possess low

10 acceptability among the researcher communities. The conventional remediation techniques

11 include pneumatic fracturing, solidification/stabilization, vitrification, excavation/removal

12 of contaminated soil layer, chemical oxidation, soil washing, chemical precipitation, ion-

13 exchange, adsorption, membrane filtration and electrochemical treatment technologies

14 (Bhargava et al., 2012; Bharti and Kumar Banerjee, 2012; Mahar et al., 2016). 
Solid Earth Discuss., https://doi.org/10.5194/se-2017-75

Manuscript under review for journal Solid Earth

Discussion started: 6 November 2017

(c) Author(s) 2017. CC BY 4.0 License.

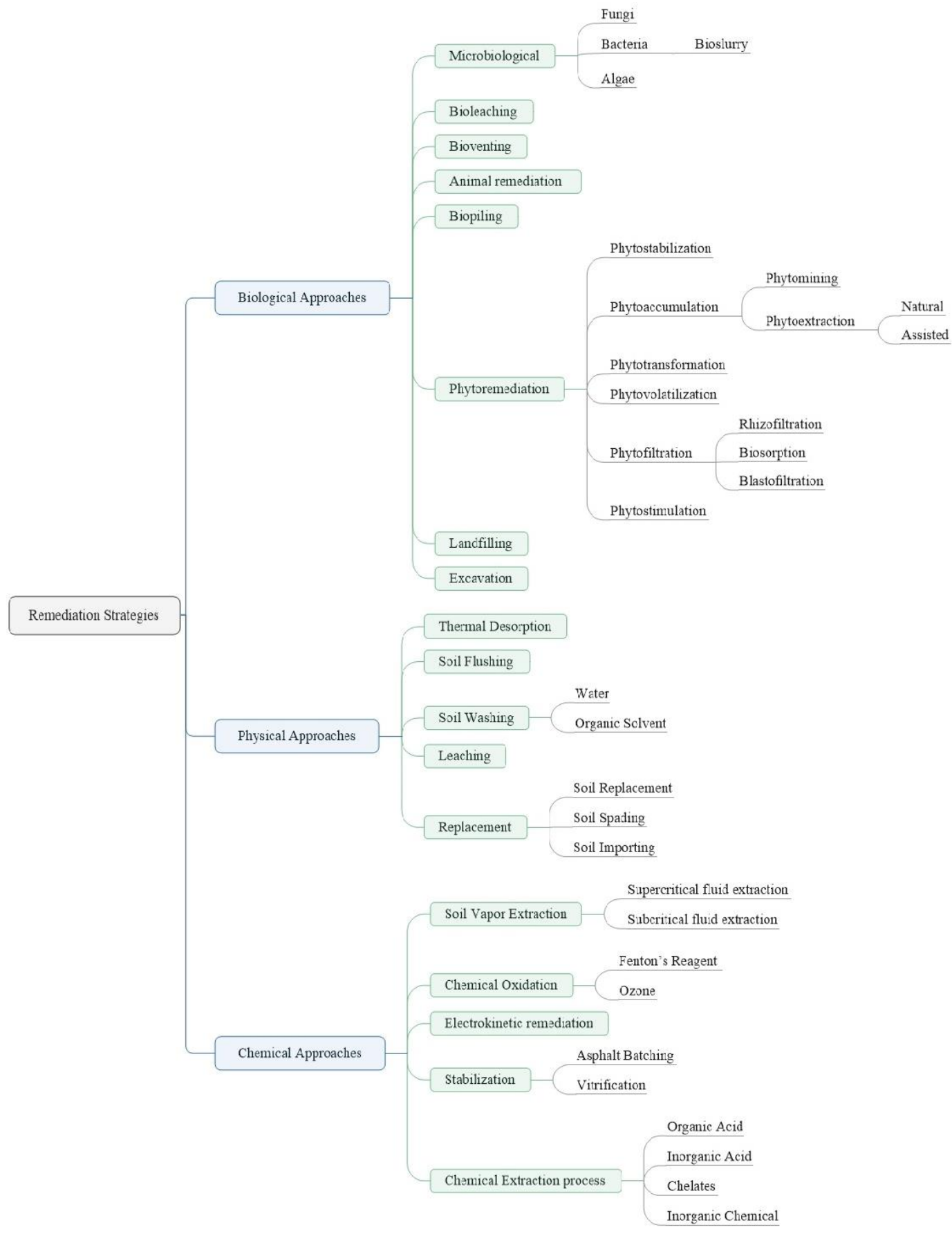

Figure 2. Different remediation strategies used for soil heavy metals treatment. 
Solid Earth Discuss., https://doi.org/10.5194/se-2017-75

Manuscript under review for journal Solid Earth

Discussion started: 6 November 2017

(c) Author(s) 2017. CC BY 4.0 License.

Phytoremediation involve the use of plants to extract, sequester, and detoxify environmental contaminants (heavy metals, radionuclides, pesticides and polychlorinated biphenyls) from soil. Phytoremediation was introduced as a new discipline in 1970s and developed with the successive discoveries of hyperaccumulators and advancement of analytical techniques in the twentieth century. The concept of moving from 'phytoremediation' to 'phytomining' to recover valuable metals for economic benefits is underway (Ha et al., 2011; Jiang et al., 2015). Phytoremediation cost 25-100 US\$ per ton, while conventional excavation/landfill cost is $150-350$ US\$. Phytoremediation is attracting the attention of research scientists, remediation experts and environmental professionals in different industrial and government sectors, due to its high potential, easiness, efficiency and economic benefits than the other technologies. Phytoremediation can simultaneously detoxify hazardous waste and helps in restoration of polluted sites (Bharti and Kumar Banerjee, 2012; Dermont et al., 2008). Phytoremediation technologies are classified as phytoextraction, phytofiltration, phytostabilization, phytovolatilization, rhizodegradation and phytodesalination (Ali et al., 2013; Ha et al., 2011; Mahar et al., 2016). Different strategies used for remediation and restoration of polluted sites are given in Table 1.

Phytoremediation provide an opportunity for food biofortification with micronutrients (Fe, $\mathrm{Zn}$ ) and ultimately provide an inorganic supplement for improving human health. Fortification of vegetables with Se gave impressive results (Banuelos, 2006). Biofortification is gaining importance, as large number of international research programs have been recently launched (Qaim et al., 2007). However, medical trials, toxicity and appropriate dosages assessment are needed before biofortified products can be distributed 
Solid Earth Discuss., https://doi.org/10.5194/se-2017-75

Manuscript under review for journal Solid Earth

Discussion started: 6 November 2017

(c) Author(s) 2017. CC BY 4.0 License.

1 and consumed (Zhao and Mcgrath, 2009). Phytoremediation can generate revenue by the

2 production of biofuels, nonconsumable agricultural products, or wood is economically

3 viable in many countries (Lehmann, 2007). Apart from biofuel, the production of metal rich

4 biochar can provide a new perspective in the remediation of contaminated sites and its

5 application as a fertilizer. The application of biochar can provide plant nutrients, improve

6 soil health, sequester carbon and mitigate climate changes. Phytoremediation provided a

7 niche for native animals and birds in Guadiamar Green Corridor programme (Evangelou

8 and Deram, 2014). Accumulation of heavy metals ( $\mathrm{Zn}$ and Ni) in plants through

9 phytoremediation provides defense against chewing insects. Phytoremediation with

10 multiple plants specie can counter the adverse soil and environmental condition (Conesa et

11 al., 2012).

Table 1. Summary of phytoremediation techniques

\begin{tabular}{|l|l|l|}
\hline Techniques & Description & References \\
\hline $\begin{array}{l}\text { Phytoextraction } \\
\text { (Phytoaccumulation) }\end{array}$ & $\begin{array}{l}\text { Accumulation of pollutants in harvestable biomass i.e., } \\
\text { shoots }\end{array}$ & (Erdei et al., 2005) \\
\hline Phytofiltration & $\begin{array}{l}\text { Sequestration of pollutants from contaminated waters } \\
\text { by plants }\end{array}$ & $\begin{array}{l}\text { (Tangahu et al., } \\
\text { 2011) }\end{array}$ \\
\hline Phytostabilization & $\begin{array}{l}\text { Limiting the mobility and bioavailability of pollutants } \\
\text { in soil by plant roots }\end{array}$ & $\begin{array}{l}\text { (Tangahu et al., } \\
\text { 2011) }\end{array}$ \\
\hline Phytovolatilization & $\begin{array}{l}\text { Conversion of pollutants to volatile form and their } \\
\text { subsequent release to the atmosphere }\end{array}$ & (Erdei et al., 2005) \\
\hline Phytodegradation & $\begin{array}{l}\text { Degradation of organic xenobiotics by plant enzymes } \\
\text { within plant tissues }\end{array}$ & $\begin{array}{l}\text { (Pulford and } \\
\text { Watson, 2003) }\end{array}$ \\
\hline $\begin{array}{l}\text { Rhizodegradation } \\
\text { (Phytotransformation) }\end{array}$ & $\begin{array}{l}\text { Degradation of organic xenobiotics in the rhizosphere } \\
\text { by rhizospheric microorganisms }\end{array}$ & (Mahar et al., 2016) \\
\hline Phytodesalination & Removal of excess salts from saline soils by halophytes & (Ali et al., 2013) \\
\hline
\end{tabular}

\section{Phytoextraction (phytoaccumulation) of $\mathrm{Cd}$ and $\mathbf{P b}$}

Phytoextraction is the uptake of contaminants from soil/water via roots and their translocation into the plant shoot, to eradicate contaminants and encourage long-term 
Solid Earth Discuss., https://doi.org/10.5194/se-2017-75

Manuscript under review for journal Solid Earth

Discussion started: 6 November 2017

(c) Author(s) 2017. CC BY 4.0 License.

1 cleanup of soil or wastewater (Bhargava et al., 2012; Mahar et al., 2016). Among different

2 strategies adopted by plants for the remediation of heavy metals from soil and water,

3 phytoextraction is publicly appealing remediation (green) technology to be practiced at

4 field level (Ali et al., 2013; Mahar et al., 2016). A heavy metal tolerant plant used for the

5 phytoextraction must be capable to grow rapidly with high biomass yield per hectare,

6 metal-hyperaccumulator and has prolific root system.

The identification and selection of appropriate hyperaccumulator plant is vital to phytoextraction process, which can accumulate exceptional concentrations of HMs in aerial parts without evident toxicity signs. Different research studies have reported more than 500 plant species (400 hyperaccumulators) including 101 families of Asteraceae, Brassicaceae, Caryophyllaceae, Cyperaceae, Cumouniaceae, Fabaceae, Flacourtiaceae, Lamiaceae, Poaceae, Violaceae and Euphobiaceae as hyperaccumulators (Bolan et al., 2014; Liu et al., 2009).

Hyperaccumulators can concentrate $>10,000 \mathrm{mg} \mathrm{kg}^{-1} \mathrm{Zn}$ and $\mathrm{Ni}, 1000 \mathrm{mg} \mathrm{kg}^{-1} \mathrm{Co}$, $\mathrm{Cu}, \mathrm{Cr}$ and $\mathrm{Pb}, 100 \mathrm{mg} \mathrm{kg}^{-1} \mathrm{Cd}, 10 \mathrm{mg} \mathrm{kg}^{-1} \mathrm{Hg}$; (2) bioconcentration factor and translocation factor > 1.0 (Ha et al., 2011; Wei et al., 2012). Metal hyperaccumulator species have attracted considerable research interest during the last four decades, because of their evident significance for cleaning contaminated soils (Rascio and Navariizzo, 2011). List of heavy metal hyperaccumulators, especially $\mathrm{Cd}$ and $\mathrm{Pb}$ is given in Table 2, cited in different scientific literature worldwide (Bech et al., 2012b; Chen et al., 2014; Deng et al., 2014; Salazar and Pignata, 2013; Zhang et al., 2014). During phytoextraction annual crops and grasses are preferred due to their short growth periods and adoptability to 
Solid Earth Discuss., https://doi.org/10.5194/se-2017-75

Manuscript under review for journal Solid Earth

Discussion started: 6 November 2017

(c) Author(s) 2017. CC BY 4.0 License.

1 environmental stress like water scarcity and high temperature (Ali et al., 2013). Literature

2 has also reported the use of field crops (maize, rice, barley, beetroot, oats, tobacco and

3 sunflower), vegetables (green onion and tomato) and trees (willow, poplar, castor oil and

4 acacia) for soil HMs extraction (He et al., 2009; Luo et al., 2005; Marmiroli et al., 2013).

5 Cadmium is present in most of the zinc contaminated sites. Different plants such as

6 Impatiens walleriana, Pteris vittata, Sedum alfredii and Thlaspi caerulescens can extract

$7 \quad 1168,6434,922.6$ and $7400 \mathrm{mg} \mathrm{kg}^{-1} \mathrm{Cd}$, respectively from soil (Escarre et al., 2000; Wei et

8 al., 2012; Wenhao et al., 2013; Xiao et al., 2008). Many plants can accumulate lead in a

9 very high concentration in its different parts. Lepidium bipinnatifidum, Thlaspi

10 rotundifolium and Zea mays can be effectively used as a hyperaccumulators for

11 contaminated soils to extract up to 7240,8200 and $10600 \mathrm{mg} \mathrm{kg}^{-1} \mathrm{~Pb}$, respectively (Bech et

12 al., 2012a; Huang and Cunningham, 1996; Reeves and Brooks, 1983). 
Solid Earth Discuss., https://doi.org/10.5194/se-2017-75

Manuscript under review for journal Solid Earth

Discussion started: 6 November 2017

(c) Author(s) 2017. CC BY 4.0 License.

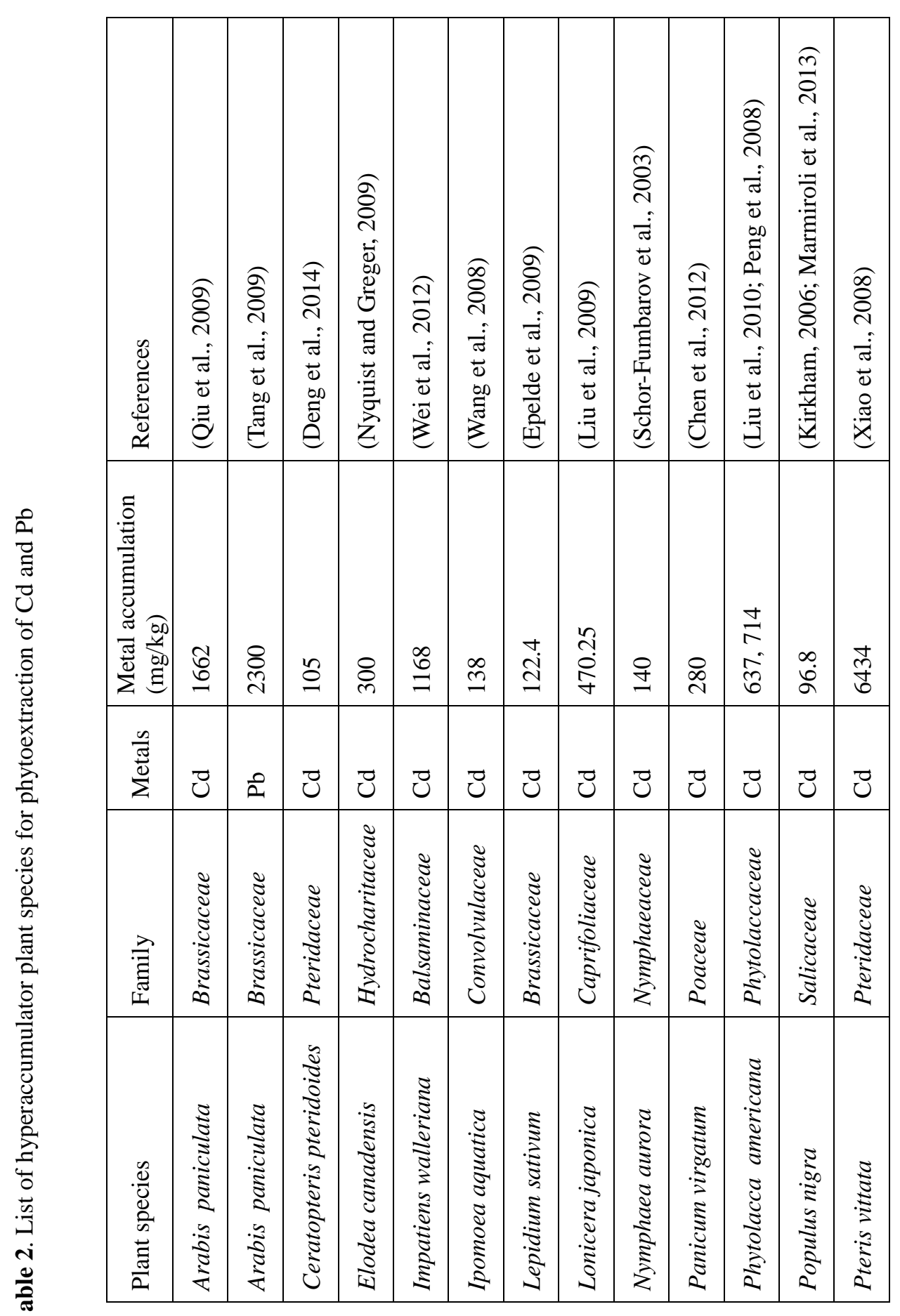


Solid Earth Discuss., https://doi.org/10.5194/se-2017-75

Manuscript under review for journal Solid Earth

Discussion started: 6 November 2017

(c) Author(s) 2017. CC BY 4.0 License.

\begin{tabular}{|c|c|c|c|c|c|c|c|c|c|c|c|c|c|c|c|}
\hline 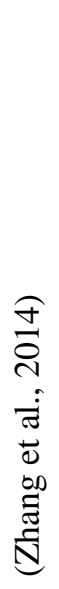 & 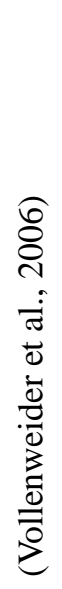 & 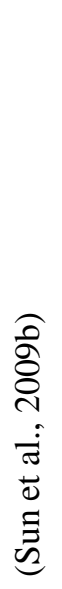 & 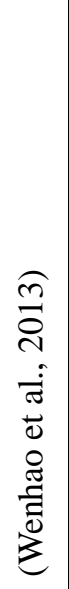 & 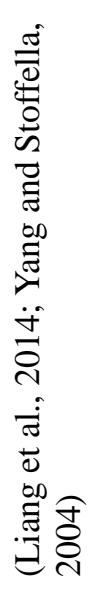 & 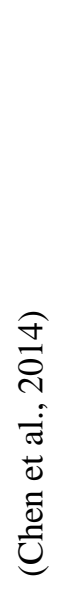 & 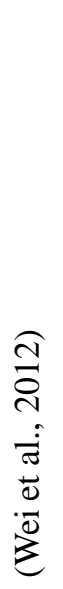 & 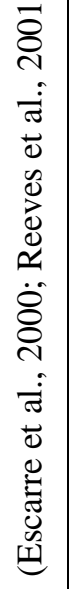 & 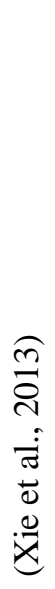 & 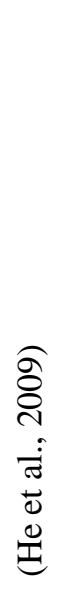 & 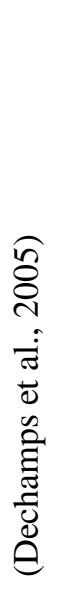 & 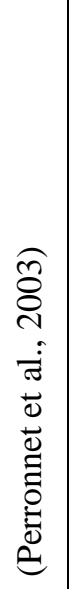 & 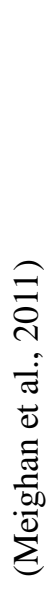 & 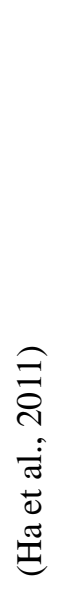 & 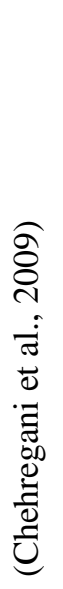 & 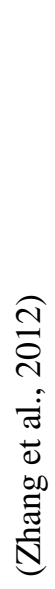 \\
\hline 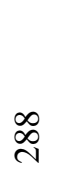 & ఠి & $\stackrel{n}{0}$ & $\begin{array}{l}0 \\
\text { Ṅ } \\
\sigma\end{array}$ & 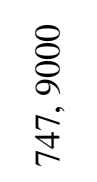 & $\stackrel{\Upsilon}{\stackrel{N}{\Xi}}$ & $\underset{\sim}{\stackrel{\sim}{2}}$ & $\begin{array}{l}8 \\
8 \\
8 \\
8 \\
8 \\
\stackrel{+}{1}\end{array}$ & 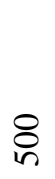 & $\begin{array}{l}\stackrel{O}{\hat{~}} \\
\dot{ \pm} \\
\text { I }\end{array}$ & 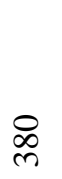 & 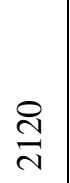 & 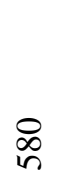 & 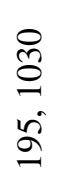 & 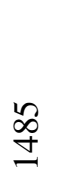 & 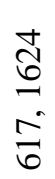 \\
\hline 己 & $\bar{u}$ & $\bar{U}$ & $\bar{U}$ & 己 & $\ddot{U}$ & $\tilde{U}$ & $\tilde{0}$ & $\tilde{U}$ & $\frac{0}{\overrightarrow{0}}$ & تే & $\overrightarrow{0}$ & $\tilde{U}$ & $\begin{array}{l}\overrightarrow{0} \\
\overrightarrow{0}\end{array}$ & $\overrightarrow{2}$ & $\begin{array}{l}\overrightarrow{0} \\
\overrightarrow{0}\end{array}$ \\
\hline 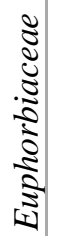 & 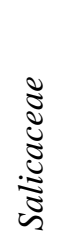 & 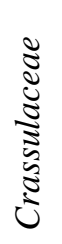 & 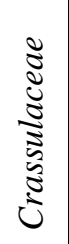 & 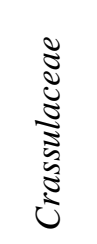 & 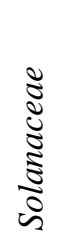 & 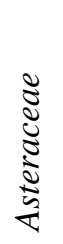 & 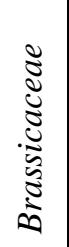 & 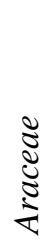 & $\begin{array}{l}\mathbb{8} \\
\mathbb{\Xi} \\
0 \\
2\end{array}$ & 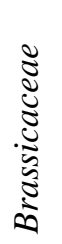 & 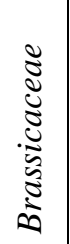 & 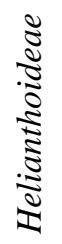 & 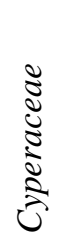 & 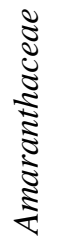 & 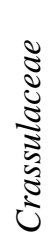 \\
\hline 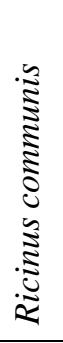 & 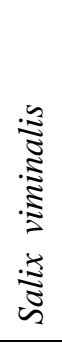 & 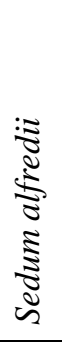 & 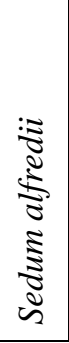 & 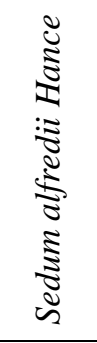 & 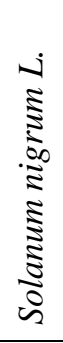 & 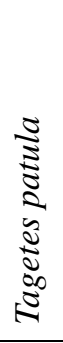 & 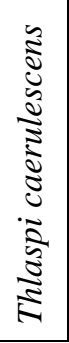 & $\begin{array}{l}0 \\
0 \\
0 \\
0 \\
0 \\
0 \\
0 \\
0 \\
0 \\
5 \\
0 \\
0\end{array}$ & $\begin{array}{l}0 \\
0 \\
0 \\
0 \\
0 \\
0 \\
0 \\
0 \\
0 \\
0\end{array}$ & 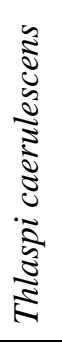 & 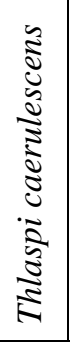 & 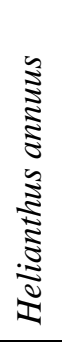 & 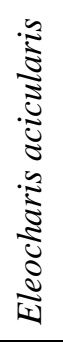 & 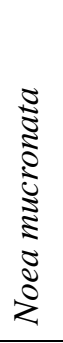 & 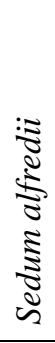 \\
\hline
\end{tabular}


Solid Earth Discuss., https://doi.org/10.5194/se-2017-75

Manuscript under review for journal Solid Earth

Discussion started: 6 November 2017

(c) Author(s) 2017. CC BY 4.0 License.

\begin{tabular}{|c|c|c|c|c|c|c|c|c|c|c|c|c|c|c|c|}
\hline 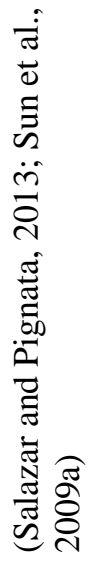 & 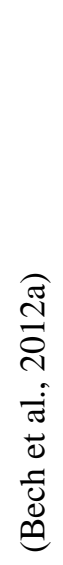 & 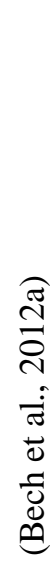 & 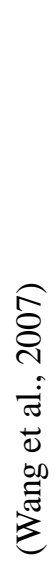 & 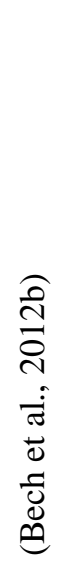 & 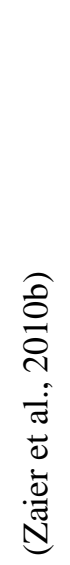 & 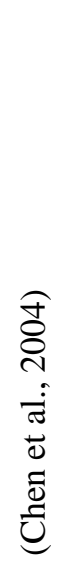 & 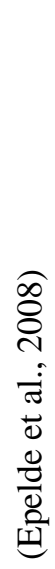 & 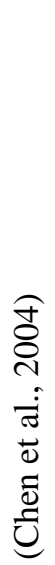 & 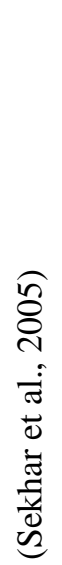 & 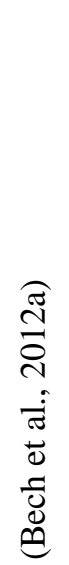 & 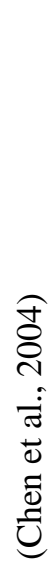 & 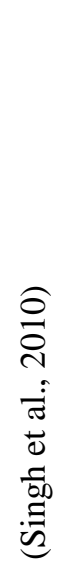 & 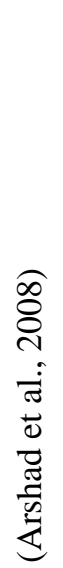 & 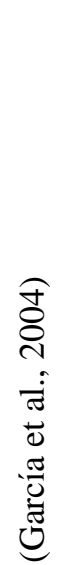 & 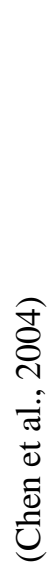 \\
\hline $\begin{array}{l}\stackrel{b}{8} \\
8 \\
\stackrel{8}{0} \\
\stackrel{8}{8}\end{array}$ & 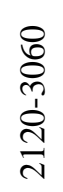 & $\begin{array}{l}8 \\
8 \\
\stackrel{2}{1} \\
\grave{1} \\
\stackrel{\infty}{\pi}\end{array}$ & $\begin{array}{l}\text { Jo } \\
\stackrel{d}{d}\end{array}$ & $\frac{\infty}{n}$ & ત્તે & $\begin{array}{l}8 \\
\stackrel{8}{1} \\
\stackrel{2}{2}\end{array}$ & ल & $\underset{\infty}{\varnothing}$ & 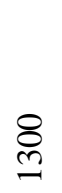 & 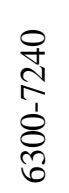 & ஓి & $\begin{array}{l}+⿱ n \\
n \\
n\end{array}$ & ষి & $\underset{\infty}{\stackrel{\infty}{\Omega}}$ & $\stackrel{\varrho}{\Xi}$ \\
\hline $\begin{array}{l}\overrightarrow{0} \\
\overrightarrow{0}\end{array}$ & $\hat{2}$ & $\overrightarrow{2}$ & $\overrightarrow{2}$ & $\hat{2}$ & $\hat{2}$ & $\hat{2}$ & $\overrightarrow{0}$ & $\overrightarrow{2}$ & $\hat{2}$ & $\hat{2}$ & $\overrightarrow{2}$ & $\hat{2}$ & $\overrightarrow{2}$ & $\hat{2}$ & $\overrightarrow{2}$ \\
\hline 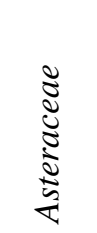 & 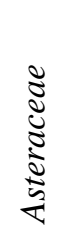 & 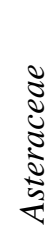 & 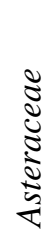 & 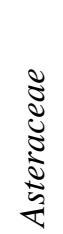 & 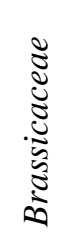 & 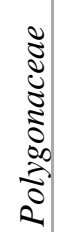 & 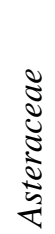 & 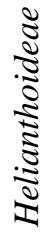 & 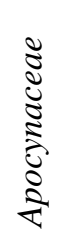 & 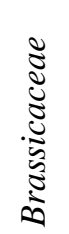 & 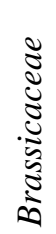 & 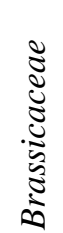 & 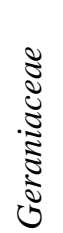 & $\begin{array}{l}\tilde{\Xi} \\
\tilde{U} \\
0 \\
0\end{array}$ & 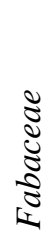 \\
\hline 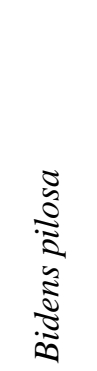 & 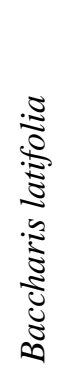 & 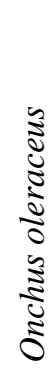 & 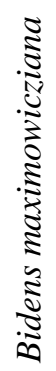 & 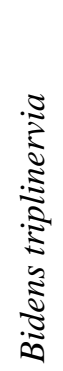 & 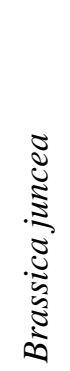 & 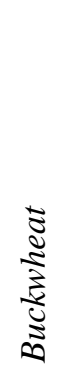 & 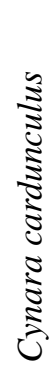 & 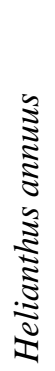 & 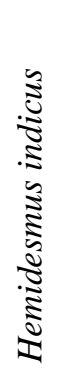 & 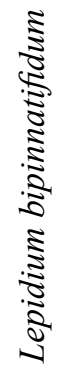 & 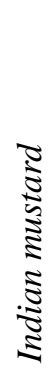 & 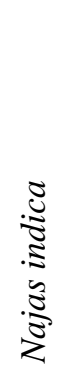 & 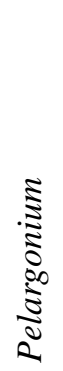 & 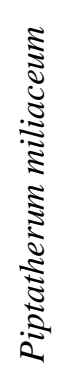 & 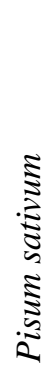 \\
\hline
\end{tabular}


Solid Earth Discuss., https://doi.org/10.5194/se-2017-75

Manuscript under review for journal Solid Earth

Discussion started: 6 November 2017

(c) Author(s) 2017. CC BY 4.0 License.

\begin{tabular}{|c|c|c|c|c|c|c|c|c|c|c|}
\hline 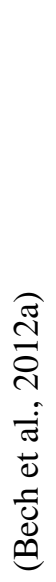 & 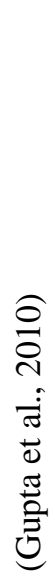 & 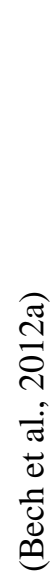 & 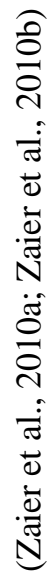 & 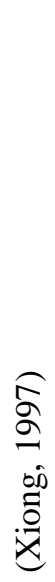 & 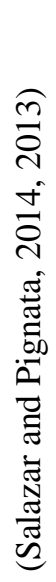 & 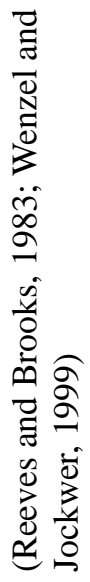 & 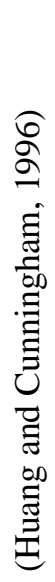 & 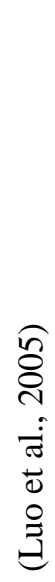 & 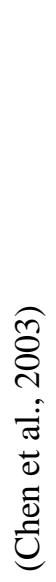 & 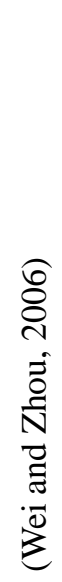 \\
\hline 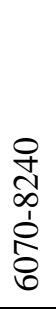 & $\begin{array}{l}\text { ¿̊ } \\
\text { ஜn }\end{array}$ & $\stackrel{\tilde{n}}{\mathfrak{y}}$ & ষ্ণ & $\stackrel{\varrho}{\Xi}$ & $\begin{array}{l}n \\
\infty \\
\infty \\
n\end{array}$ & $\begin{array}{l}\infty \\
\stackrel{\infty}{0} \\
\stackrel{\infty}{\delta} \\
\stackrel{\infty}{0}\end{array}$ & $\begin{array}{l}8 \\
8 \\
8\end{array}$ & $\begin{array}{l}\overrightarrow{0} \\
\dot{0} \\
\dot{\infty}\end{array}$ & 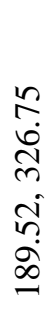 & $\overrightarrow{\tilde{n}}$ \\
\hline $\overrightarrow{2}$ & $\hat{2}$ & $\hat{2}$ & $\hat{2}$ & $\hat{2}$ & $\hat{2}$ & $\begin{array}{l}\vec{u} \\
\hat{0}\end{array}$ & $\hat{2}$ & $\begin{array}{l}\overrightarrow{0} \\
\hat{0}\end{array}$ & $\begin{array}{l}\overrightarrow{0} \\
\hat{0}\end{array}$ & $\bar{U}$ \\
\hline 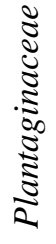 & 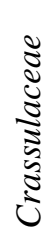 & 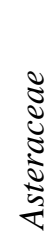 & 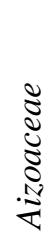 & 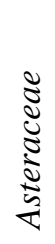 & 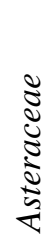 & 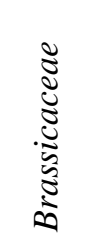 & $\begin{array}{l}\tilde{\Xi} \\
\mathbb{Z} \\
\mathbb{0} \\
2\end{array}$ & $\begin{array}{l}\tilde{E} \\
\tilde{\Xi} \\
\mathbb{8} \\
\mathbb{8}\end{array}$ & 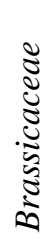 & 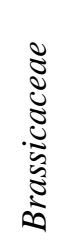 \\
\hline 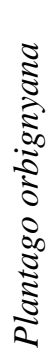 & 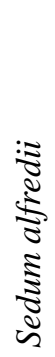 & 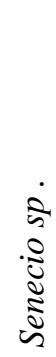 & 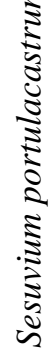 & 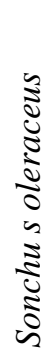 & 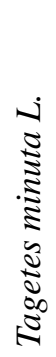 & 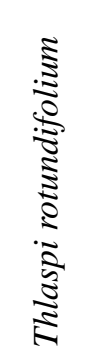 & 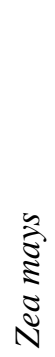 & 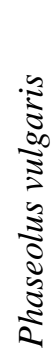 & 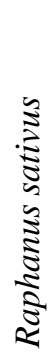 & $\begin{array}{l}0 \\
0 \\
0 \\
0 \\
0 \\
0 \\
0 \\
0 \\
0\end{array}$ \\
\hline
\end{tabular}

ร 
Solid Earth Discuss., https://doi.org/10.5194/se-2017-75

Manuscript under review for journal Solid Earth

Discussion started: 6 November 2017

(c) Author(s) 2017. CC BY 4.0 License.

1

2

4

5

\section{Induced and natural phytoextraction of $\mathrm{Cd}$ and $\mathrm{Pb}$}

Naturally plants can extract lower concentration of eavy metals from the soil solution and this capacity can be improved by introduction of chelates and complexing agents. Phytoextraction can be induced (chelate assisted) or natural (continuous). Induced phytoextraction is driven by chelates, while, natural phytoextraction is based on the hyperaccumulators with no soil amendments (Hseu et al., 2013; Liang et al., 2014;

Saifullah et al., 2010; Schor-Fumbarov et al., 2003).

Chelant-enhanced phytoextraction is cost-effective substitute to conventional techniques for soil HMs remediation. Besides mobilizing metals in soil, chelates also facilitate metal translocation from root to shoot. Chelates help in HMs desorption from soil particles and form metal-chelant complexes in soil, drawn upward by passive apoplastic pathway. The use of chelates is reported in various phytoextraction studies (Epelde et al., 2008; Evangelou et al., 2006; Liang et al., 2014; Zhang et al., 2014), where it enhanced the capability of hyperaccumulator plants to extract higher quantity of $\mathrm{HMs}(\mathrm{Cd}, \mathrm{Pb})$ from the soil-water system (Freitas et al., 2013; Hadi et al., 2010; Saifullah et al., 2010). The Pb uptake is not improved to the required level by the chelates application. The main reason is supposed to be the root injury caused by chelates. While, the other metals uptake is improved by chelates application in field trials. However, chelates can cause secondary pollution. The excess use of EDTA increase the risk of leaching metallic ions from the soil to groundwater causing severe health hazards and ill effects on the plant biomass and growth (Evangelou et al., 2008). 
Solid Earth Discuss., https://doi.org/10.5194/se-2017-75

Manuscript under review for journal Solid Earth

Discussion started: 6 November 2017

(c) Author(s) 2017. CC BY 4.0 License.

Natural chelating agents like EDDS and nitrilotriacetic acid (NTA) can be an alternate for EDTA. But it also has leaching and toxicity effects on plants. Thus, proper care should be taken when practicing induced phytoextraction (Evangelou et al., 2008; Song et al., 2012). At phytotoxic level of metals in the soil, lime and organic matter can be a best choice for delaying solubility (Pilonsmits, 2005). The use of citric acid as a chelating agent could be promising, because it has a natural origin and is easily biodegraded in soil. Furthermore, citric acid is nontoxic to plants, therefore plant growth is not restricted (Smolińska and K, 2007). Chelates can be particularly useful in mobilizing heavy metals at high soil $\mathrm{pH}$ as the stability of metal-organic complex increases with increasing $\mathrm{pH}$. The common chelates used for enhancing the $\mathrm{HMs}(\mathrm{Cd}, \mathrm{Pb})$ phytoextraction are presented in

Table 3.

Table 3: List of chelates used for inducing $\mathrm{Cd}$ and $\mathrm{Pb}$ uptake by hyperaccumulators

\begin{tabular}{|l|l|l|}
\hline Chelates or complexing agents & Metals assisted & References \\
\hline EDDS and NTA & $\mathrm{Cd}$ & (Hseu et al., 2013) \\
\hline Humic acid, EDTA & $\mathrm{Cd}$ & $\begin{array}{l}\text { (Evangelou et al., 2004; Schor- } \\
\text { Fumbarov et al., 2003) }\end{array}$ \\
\hline Elemental sulfur, EDTA & $\mathrm{Cd}, \mathrm{Pb}$ & $\begin{array}{l}\text { (Liang et al., 2014; Saifullah et al., } \\
\text { 2010) }\end{array}$ \\
\hline Citric acid & $\mathrm{Cd}, \mathrm{Pb}$ & (Freitas et al., 2013; Gao et al., 2012) \\
\hline EDTA & $\mathrm{Pb}, \mathrm{Cd}$ & $\begin{array}{l}\text { (Gabos et al., 2009; Wang et al., 2007; } \\
\text { Wei et al., 2012; Zhang et al., 2014) }\end{array}$ \\
\hline EDDS, MGDA & $\mathrm{Pb}$ & (Cao et al., 2007) \\
\hline EDTA, EDDS & $\mathrm{Pb}, \mathrm{Cd}$ & (Meers et al., 2007) \\
\hline EDTA, PDTA & $\mathrm{Pb}$ & (Cho et al., 2009) \\
\hline EDTA, EDDS & $\mathrm{Pb}, \mathrm{Cd}$ & (Luo et al., 2005) \\
\hline EDTA and EDDS & $\mathrm{Pb}$ & (Chen et al., 2004; Epelde et al., 2008) \\
\hline Na2-EDTA & $\mathrm{Pb}$ & (Evangelou et al., 2006) \\
\hline HEDTA & $\mathrm{Pb}$ & (Huang and Cunningham, 1996) \\
\hline Citric acid $(\mathrm{CA})$ & $\mathrm{Pb}, \mathrm{Cd}$ & (Chen et al., 2003) \\
\hline & & \\
\hline
\end{tabular}


Solid Earth Discuss., https://doi.org/10.5194/se-2017-75

Manuscript under review for journal Solid Earth

Discussion started: 6 November 2017

(c) Author(s) 2017. CC BY 4.0 License.

2 following characteristics (Ali et al., 2013; Bhargava et al., 2012; Mahar et al., 2016).

3

4

5

6

7

8

9 Massive growth potential and high biomass production.

(i) Extensive root system and root developing capacity in adverse condition.

(ii) Ability to grow outside their area of collection.

(iii) Higher accumulation rate of target heavy metals from soil and translocation of the accumulated heavy metals from roots to shoots for successful phytomining.

(iv) Tolerance to the toxic effects of the target heavy metals.

(v) Good adaptation to prevailing environmental and climatic conditions (drought, temperature, humidity, salinity, nutrient deficiency and water logging).

(vi) Easy cultivation, harvest and resistance to pathogens and pests attack.

(vii) Repulsion to herbivores to avoid food chain contamination.

Phytoextraction is an income-generating, solar driven technology, removing precious metals from the soil as bio-ore, generate energy through biomass burning i.e., phytomining (Brooks and Robinson, 1998; Ha et al., 2011; Li et al., 2003). Phytoremediation is the stabilization or recovery of metal contaminants for secure disposal, while phytomining refers to the recovery of precious metals $(\mathrm{Au}, \mathrm{Pt}, \mathrm{Ni}$ and $\mathrm{Tl})$ via growing hyperaccumulators for monetary return (Mcgrath and Zhao, 2003).

\section{Phytomining of heavy metals}

It is an environment friendly technology of growing metal hyperaccumulator plants, harvesting the biomass and burning it to produce a bio-ore as shown in Figure 3 (Ha et al., 
Solid Earth Discuss., https://doi.org/10.5194/se-2017-75

Manuscript under review for journal Solid Earth

Discussion started: 6 November 2017

(c) Author(s) 2017. CC BY 4.0 License.

1 2011). Phytomining offers the possibility of exploiting ores/mineralized soils that are not

2 economic to explore by conventional techniques. The metal content of bio-ore is greater

3 than conventional ore and requires less storage space due to low density. Moreover,

4 phytomining is an environmentally responsible approach to site remediation. A well-

5 planned phytoremediation/phytomining operation will result in commercially viable metal-

6 enriched bio-ore. The mined soil can be used for agriculture (forestry and horticulture) and

7 commercial use (Sheoran et al., 2009). Research efforts are underway to recognize the

8 economic potential of this green technology. Practicing phytomining will hamper the

9 distribution of HMs by surface runoff and wind, reduce leaching into aquifers, provide

10 vegetation to control water and wind erosion of soil. Phytomining is considered an aesthetic,

11 safe and nondestructive technology, with high public and commercial acceptance (Sheoran

12 et al., 2009).

13 The pioneering field trial for phytomining was reported by the US Bureau of the Mines,

Reno, Nevada on a naturally occurring strain of Streptanthus polygaloides which is a specie

known to hyperaccumulate nickel (Chaney et al., 1998). Several plant species are renowned

(Anderson et al., 2005; Boominathan et al., 2004). Phytomining not only provide precious

Robinson, 1998). Phytomining is less intrusive, requires less energy than traditional mining

technology. Phytomining has minimal environmental disturbances and effects due to 
Solid Earth Discuss., https://doi.org/10.5194/se-2017-75

Manuscript under review for journal Solid Earth

Discussion started: 6 November 2017

(c) Author(s) 2017. CC BY 4.0 License.

1 by opencast mining operation (Robinson and Mcgrath, 2003). Vegetation cover can

2 stabilize and accelerate ecological succession (Sheoran et al., 2009).

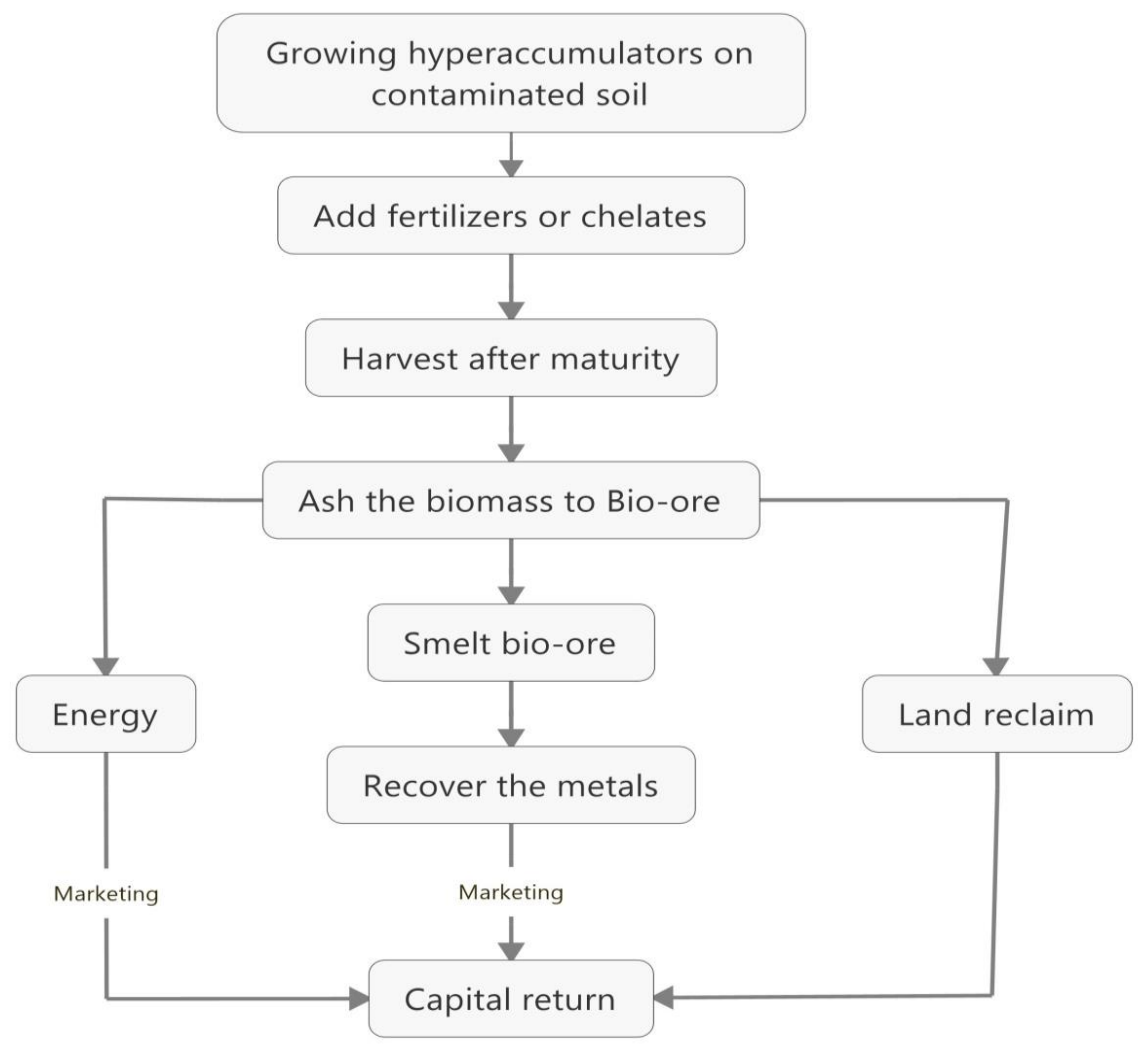

3

4 Figure 3. An illustrative model of economical phytomining of heavy metals.

$5 \quad$ Phytomining faces similar limitations as phytoextraction process, like soil $\mathrm{pH}$, climatic

6 conditions, root depth, solubility and availability of HMs and nutrients affecting plant

7 growth (Li et al., 2003). Phytomining can assist in generating revenue along with

8 rehabilitation and sustainable closure of mining sites (Wilson-Corral et al., 2011). 
Solid Earth Discuss., https://doi.org/10.5194/se-2017-75

Manuscript under review for journal Solid Earth

Discussion started: 6 November 2017

(c) Author(s) 2017. CC BY 4.0 License.

1

2

3

4

5

\section{An insight into economics of heavy metals phytomining}

Some reclaimed metals ( $\mathrm{Tl}, \mathrm{Au}, \mathrm{Co}, \mathrm{Ni}, \mathrm{Cu}, \mathrm{U}, \mathrm{Cd}, \mathrm{Zn}, \mathrm{Pb}, \mathrm{Mn}$, and $\mathrm{Se}$ ) may provide additional revenue by phytomining (Thangavel and Subbhuraam, 2004). The remediation market around the world is estimated to be nearly 34-54 billion US\$ (Evangelou and Deram, 2014). Several companies and scientific research groups are pursuing phytomining strategies. Berkheya coddii, Daucus carota and Brassica juncea are reported to accumulate as much as $20 \mathrm{mg} \mathrm{kg}^{-1}$ of gold after ammonium thiocyanate supplementation (Prasad, 2003).

Some companies are gaining profit not just by recovering metals from the biomass, but also using the biomass for energy generation and the ash as a source of carbon and potash as well as gaining benefits from the sale of carbon dioxide credits (Rosenfeld and Henry, 2001; Sheoran et al., 2009). Research showed the extraction of highly pure $\mathrm{Ni}$ from $\mathrm{Ni}$ contaminated Alyssum biomass, which can be used as substitute for Ni fertilizer. A number of phytomining companies have emerged in US, Canada, Western and Eastern Europe, Japan, Australia, Latin America and an emerging market also exists in Asia (China).

The economics of phytomining is influenced by a number of factors, i.e., the metal content in soil and plant, annual biomass production and whether the energy of combustion of the biomass can be recovered and sold. The biomass production plays an important role in adaptation of hyperaccumulator for phytomining operation in future agrofarming. The most important factor, however, is worldwide price of metal being phytomined (Brooks and Robinson, 1998; Harris et al., 2009). Metal value ranges from $\$ 1.793$ to $\$ 39368.59 \mathrm{~kg}^{-1}$ for lead and gold, respectively (March, 2016 shown in Table 4). The best candidate metals for phytomining are $\mathrm{Au}, \mathrm{Tl}, \mathrm{Co}$, and $\mathrm{Ni}$ due to their high market prices and metal concentration 
Solid Earth Discuss., https://doi.org/10.5194/se-2017-75

Manuscript under review for journal Solid Earth

Discussion started: 6 November 2017

(c) Author(s) 2017. CC BY 4.0 License.

1 in biomass of hyperaccumulators. Though, the price of uranium and gold are comparatively

2 high among the candidate metals, but its reported metal concentration $\left(100,10 \mathrm{mg} \mathrm{kg}^{-1}\right)$ in

3 biomass $\left(10000,20000 \mathrm{~kg} \mathrm{ha}^{-1}\right)$ is low, which makes Atriplex confertifolia, Berkheya coddii

4 it uneconomical for phytomining (Mahar et al., 2016; Sheoran et al., 2009). The high

5 market value can compensate to some extent the low biomass, but low biomass can reduce

6 the yield of the metal in the bio-ore and hence reduce the profit. The price of Mn was low

$7 \quad\left(\$ 1.91 \mathrm{~kg}^{-1}\right)$ but plant concentration $\left(1650 \mathrm{mgkg}^{-1}\right)$ was high in Macadamia neurophylla,

8 making it more practical than Haumaniastrum katangense and Atriplex confertifolia used

9 for Copper and Uranium, respectively (Jaffré, 1980). Metals prices are subjected to global

10 economics condition and current low/high value of a metal cannot ensure its consideration

11 for permanent phytomining. The produced biomass could be combusted to ash, stored until

12 the world price hikes (Brooks and Robinson, 1998). Reviewing the published scientific

13 literature, the plant species reported for phytoextraction of precious metals ( $\mathrm{Tl}, \mathrm{Au}, \mathrm{Co}, \mathrm{Ni}$,

$14 \mathrm{Cu}, \mathrm{U}, \mathrm{Cd}, \mathrm{Zn}, \mathrm{Pb}, \mathrm{Mn}$, and $\mathrm{Se}$ ) may be used for phytomining purpose after field trials. The

15 revenue (US\$) to the grower is presented in Table 4 at the harvest time and at current, based on the price of the metals (March, 2016). 
Solid Earth Discuss., https://doi.org/10.5194/se-2017-75

Manuscript under review for journal Solid Earth

Discussion started: 6 November 2017

(c) Author(s) 2017. CC BY 4.0 License.

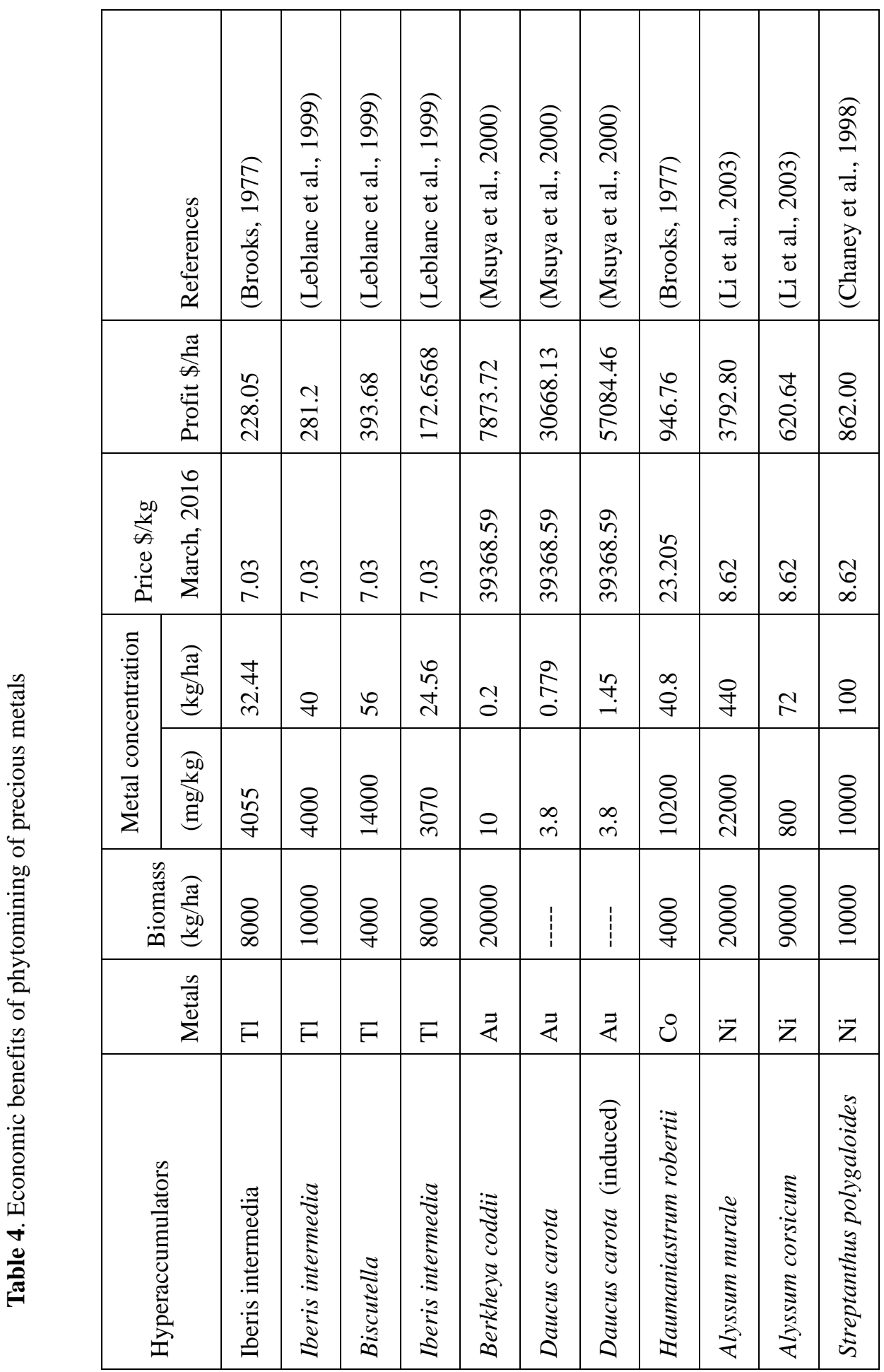


Solid Earth Discuss., https://doi.org/10.5194/se-2017-75

Manuscript under review for journal Solid Earth

Discussion started: 6 November 2017

(c) Author(s) 2017. CC BY 4.0 License.

\begin{tabular}{|c|c|c|c|c|c|c|c|c|c|}
\hline 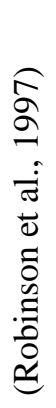 & 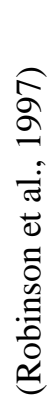 & 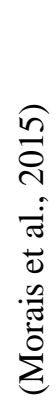 & 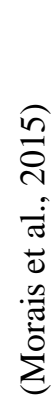 & 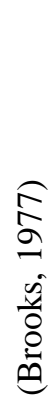 & 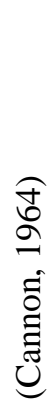 & 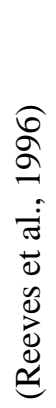 & 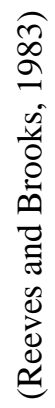 & 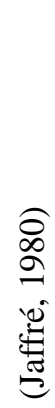 & 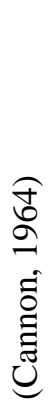 \\
\hline $\begin{array}{l}\text { Uా } \\
\stackrel{\text { }}{ } \\
\text {. }\end{array}$ & 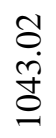 & $\begin{array}{l}\text { స్ } \\
\text { } \\
\text { ñ }\end{array}$ & 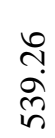 & $\underset{\nabla}{\vec{\nabla}}$ & 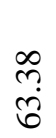 & 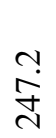 & $\begin{array}{l}\bar{\infty} \\
\infty \\
\infty\end{array}$ & $\frac{n}{n}$ & $\begin{array}{l}\infty \\
\stackrel{0}{ \pm} \\
\dot{ \pm}\end{array}$ \\
\hline $\begin{array}{l}\widetilde{W} \\
\infty\end{array}$ & $\begin{array}{l}\sigma \\
\infty \\
\infty\end{array}$ & $\begin{array}{l}\widetilde{\sigma} \\
\infty\end{array}$ & $\begin{array}{l}\widetilde{W} \\
\infty\end{array}$ & $\begin{array}{l}\text { o } \\
\dot{n}\end{array}$ & $\begin{array}{l}\text { D̃ } \\
\text { } \\
\text { } \\
\sigma\end{array}$ & $\begin{array}{l}o \\
\text { i }\end{array}$ & 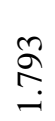 & $\bar{\sigma}$ & $\begin{array}{l}\infty \\
\stackrel{0}{ \pm} \\
\dot{J}\end{array}$ \\
\hline $\mathbb{N}$ & $\bar{\beth}$ & $\frac{n}{6}$ & $\begin{array}{l}n \\
n \\
\tilde{\sigma}\end{array}$ & $\frac{\infty}{\underset{\gamma}{\gamma}}$ & - & $\simeq$ & $\begin{array}{l}\infty \\
\text { i }\end{array}$ & $\underset{6}{0}$ & ల) \\
\hline$\underset{\infty}{\infty}$ & $\begin{array}{l}8 \\
\stackrel{n}{n} \\
n\end{array}$ & $\frac{n}{n}$ & ஸ̊ํ & $\begin{array}{l}0 \\
\infty \\
\infty\end{array}$ & 8 & 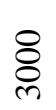 & 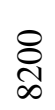 & $\begin{array}{l}8 \\
8 \\
n \\
n\end{array}$ & 8 \\
\hline ஜ & $\begin{array}{l}8 \\
\text { ¿̊ } \\
\text { ते }\end{array}$ & $\frac{\text { న }}{2}$ & $\underset{\infty}{\stackrel{\infty}{\infty}}$ & 8) & 8 & \& & \& & ষ্ঠ & \& \\
\hline $\bar{z}$ & $\bar{z}$ & $\bar{z}$ & $\bar{z}$ & $\bar{U}$ & $\triangleright$ & $\bar{v}$ & $\hat{\imath}$ & $\Sigma_{\Sigma}^{\Xi}$ & $\ddot{\sim}$ \\
\hline 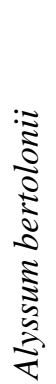 & 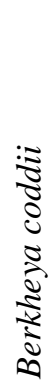 & 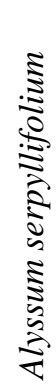 & 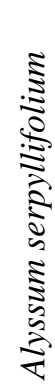 & 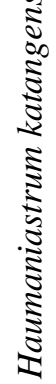 & 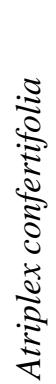 & 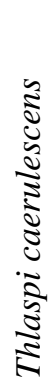 & 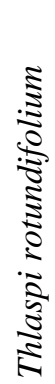 & 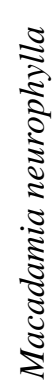 & 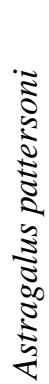 \\
\hline
\end{tabular}


Solid Earth Discuss., https://doi.org/10.5194/se-2017-75

Manuscript under review for journal Solid Earth

Discussion started: 6 November 2017

(c) Author(s) 2017. CC BY 4.0 License.

1

2

3

4

5

6

7

8

9

\section{Factors affecting phytoextract on (phytomining)}

The efficiency of hyperaccumulator plants used in phytoextraction of HMs depend on the favorable soil and environmental factors; like salinity, $\mathrm{pH}$, nutrients deficiency, HMs toxicity, speciation and bioavailability, flooding, temperature, humidity, water logging, desiccation and resistant to drought conditions (Ali et al., 2013).

The increase in clay content (clay type specially and surface area) has a negative impact on the mobility and availability of metals in soil due to fixation in clay matrix and the uptake is also pH dependent (Saifullah et al., 2010). The exchangeable and soil solution pool of metals is considered to be readily available for plant uptake (Meers et al., 2007). pH and organic matter are two of the most important soil factors that control $\mathrm{Cd}$ availability (Kirkham, 2006). Bioavailability of the heavy metals increases at low soil pH, since metal salts are soluble in acidic media. In acidic soils, metal desorption from soil binding sites into solution is stimulated due to $\mathrm{H}^{+}$competition for binding sites. Soil $\mathrm{pH}$ affects not only metal bioavailability, but also every process of metal uptake into roots. This effect appears to be metal specific. For example, in Thlaspi caerulescens, $\mathrm{Zn}$ uptake in roots showed small $\mathrm{pH}$ dependence, whereas uptake of $\mathrm{Mn}$ and $\mathrm{Cd}$ was more dependent. The CEC is a function of the amount and types of organic matter and clay minerals in the soil. The uptake of $\mathrm{Cd}$ by wheat was highest in plants grown in soils with a low CEC and vice versa. Apparently, in the soil with a high $\mathrm{CEC}$, more $\mathrm{Cd}$ was adsorbed to the exchange complexes, and hence, less Cd was available for uptake by the wheat plants. In general, sorption to soil particles reduces the activity of metals in the system. Thus, the higher the cation exchange capacity (CEC) of the soil, the greater the sorption and immobilization of the metals. 
Solid Earth Discuss., https://doi.org/10.5194/se-2017-75

Manuscript under review for journal Solid Earth

Discussion started: 6 November 2017

(c) Author(s) 2017. CC BY 4.0 License.

1

2

3

5

6

7 The major limitations of most metal phytoextraction processes are:

8

\section{Limitations of phytoextraction (Phytomining)} et al., 2012; Mahar et al., 2016).

- Bioavailability of only target metal(s).

- Plants accumulate metals within above ground biomass, which is low.

- Polluted site must be large enough to carry out phytomining.

- Extended time for remediation process.

- $\quad$ Limited to low and medium metal contaminant concentrations.

- Climate dependent/variable; seasonal effectiveness.

- Risk of metals transfer by food chain (to animals or air).

- Introduction of non-native species may affect biodiversity (competition/allelopathy). leading to ground water pollution.

- The contaminants must be in the root zone (rhizosphere) to be drawn up by plants.

- Most of the hyperaccumulators are not suitable for field applications due to low biomass and slow growth.

Although the remediation of heavy metals is effective by hyperaccumulators, but the process is limited by biogeochemical factors viz. rhizobiological activity, exudates release, prevailing temperature, soil moisture and $\mathrm{pH}$, competing ions affecting plant growth and solubility and availability of the metals in the soil-water system (Ali et al., 2013; Bhargava

- Tightly bound fraction of metals in soil clay requires higher chelate application rates, 
Solid Earth Discuss., https://doi.org/10.5194/se-2017-75

Manuscript under review for journal Solid Earth

Discussion started: 6 November 2017

(c) Author(s) 2017. CC BY 4.0 License.

1

2

4

5

\section{Conclusion and Recommendations}

The growing world population requires more food, infrastructure, transportation and industrial growth to meet their daily requirements. These activities will intensify the use of agro-chemicals in the agriculture sector, exploration of mining sites for energy and infrastructure, manufacturing of automobile for public transportation and production of households in the coming years. As a result, these activities will contribute to higher metal release into soil, air and water, leading to environmental pollution. All the known conventional remediation technologies for HMs have secondary pollution. An environment friendly and green technology known as "phytoremediation" for in situ remediation of polluted sites is easy, economical and compatible alternate to conventional technologies. Effective phytoremediation (phytoextraction) depends on phytoavailable portion of metals in soil solution, metal uptake in plant tissue and plant biomass. The metal ions are present in soil solution but the plant option for specific ion reduces the uptake capacity of plants. Metals like $\mathrm{Pb}$ can form carbonates, hydroxides and phosphates in soil and thus reduces the phytoextraction efficiency, making the natural process difficult to continue. Phytoextraction (phytomining) depends on environmental and soil properties with some limitations, like low biomass and slow growth of hyperaccumulators. But still, progressive as compared to conventional methods, as it is solar driven, low secondary pollution, hyperaccumulators used as fuel and maintain the greenery of environment. Phytomining not only generates revenue for the grower but also provides mineral supplementation and biofuel as well as increases soil health and mitigate climate changes.

Based on the previous studies the following recommendations can be made. 
Solid Earth Discuss., https://doi.org/10.5194/se-2017-75

Manuscript under review for journal Solid Earth

Discussion started: 6 November 2017

(c) Author(s) 2017. CC BY 4.0 License.

1 (i) Further exploration of hyperaccumulator plants for enhanced phytoextraction of heavy

2

3

4

5

6

7 metals is needed.

(ii) The establishment of hyperaccumulators seed bank must be encouraged, for the expansion of phytoextraction/phytomining studies in different ecological zones. The findings at different ecological zones will help in further understanding of phytoextraction/phytomining for the remediation of pollutants.

(iii) Extensive and precise research is required in the application of chelates assisted phytoextraction in order to reduce secondary pollution of soil and air.

(iv) Experimentation on cost to benefit ratios (economics) and time consumption is required to reach a final conclusion.

(v) The use of constructed wetland for improving water quality by practicing phytoextraction is required.

(vi) Molecular studies on the mechanisms of hyperaccumulation, translocation, distribution, tolerance and sensitivity of heavy metals in different plants need further attention.

(vii) Molecular techniques for the gene identification and introduction into the desired plants for effective phytoextraction.

(viii) The extraction of metals in the target sites during the phytomining need special considerations to trafficking and toxicity of heavy metals through food chain from water, soil, plant and animal to human.

(ix) Need further studies on the rhizosphere for the enhanced phytoextraction.

(x) Biofortification of vegetables with micronutrients requires authentic medical trials, precise toxicity assessment and appropriate dosages prescription. 
Solid Earth Discuss., https://doi.org/10.5194/se-2017-75

Manuscript under review for journal Solid Earth

Discussion started: 6 November 2017

(c) Author(s) 2017. CC BY 4.0 License.

1 (xi) The conversion of biomass produced by hyperaccumulator plants into biofuel and

2 biochar need investment and technical experience to meet the economic requirements.

3 (xii) Measurements for protection and conservation of native plant diversity before

$4 \quad$ introduction of alien plants for phytomining.

\section{11. Future Perspective}

6 Phytoremediation is a slow and time consuming process. Since hyperaccumulators have

7 low biomass and can extract minute quantity of HMs from the soil, which doesn't meet the

8 remediation requirements on large scale within a short time span. It can be improved by

9 exploration of fast growing plants, which yield high biomass and extract high concentration

of HMs. Plant species with short growth period, capable of rotation and resistant to

environmental stress should be identified for effective phytoextraction. Assisted

phytoextraction can be possible cost effective commercial technology for phytomining of

HMs in future, which can enhance metal uptake and reduces the environmental risks and

time for remediation process. In order to solve the problem of low solubility, soil $\mathrm{pH}$ and

fixation in clay, new research dimensions with respect to rhizosphere should be explored.

Exploration of plant growth regulators (cytokinins, gibberellic acid, indolebutyric acid, naphthylacetic acid and indole-3-acetic acid) and rhizobacteria (P solubilizing) provide a new research area with respect to the mechanism of HMs uptake and stabilization for a safe and green environment. The role of biotechnology and genetic engineering for improving the phytomining can't be ignored. Many genes are involved in metal accumulation, translocation and sequestration. Gene transfer into candidate plant is a possible strategy for genetic engineering of plants. Selection of individuals with genetic coding for high metal 
Solid Earth Discuss., https://doi.org/10.5194/se-2017-75

Manuscript under review for journal Solid Earth

Discussion started: 6 November 2017

(c) Author(s) 2017. CC BY 4.0 License.

content, high biomass production and superior tolerance to soil heavy metal content will augment metal crops. The isolation of genetic materials may allow the genetic manipulation of high biomass plants such as Zea mays, to produce a plant that will extract large quantities of metals. Genetic engineering is currently being used to improve metal hyperaccumulation

in plants by changing oxidation state of metals, enhancing metal transporters and chelators, encoding metal sequestration proteins i.e., MTs and PCs (metallothioneins and phytochelatins), transport proteins such as ZIP family proteins (zinc-iron permease) and ZAT ( $\mathrm{Zn}$ transporter). Environment friendly and biodegradable chelates should be developed. If phytomining proceed beyond the theoretical and pilot stage. Plants can be harvested and feedstock can be used for incineration. This could supply steam for electricity production. Biofortification of food and feed will meet the nutritional requirements of human and animals. Production of biofuel and metal rich biochar provide a new research area for soil nutritionist and economist in future. Before phytoremediation is fully commercialized, further research is needed to assure that tissues of plants used for phytoremediation do not have adverse environmental effects if eaten by wildlife or human. Further, explorations of efficient hyperaccumulator that produce more biomass stress the need for commercial smelting to extract the metals from plant biomass. 
Solid Earth Discuss., https://doi.org/10.5194/se-2017-75

Manuscript under review for journal Solid Earth

Discussion started: 6 November 2017

(c) Author(s) 2017. CC BY 4.0 License.

References

Ali, H., Khan, E., and Sajad, M. A.: Phytoremediation of heavy metals-Concepts and applications, Chemosphere, 91, 869-881, 2013.

Anderson, C., Moreno, F., and Meech, J.: A field demonstration of gold phytoextraction technology, Minerals Engineering, 18, 385-392, 2005.

Arain, M. B., Kazi, T. G., Jamali, M. K., Jalbani, N., Afridi, H. I., and Baig, J. A.: Speciation of heavy metals in sediment by conventional, ultrasound and microwave assisted single extraction methods: A comparison with modified sequential extraction procedure, Journal of Hazardous Materials, 154, 998-1006, 2008.

Arias-Estévez, M., López-Periago, E., Martínez-Carballo, E., Simal-Gándara, J., Mejuto, J.-C., and García-Río, L.: The mobility and degradation of pesticides in soils and the pollution of groundwater resources, Agriculture, Ecosystems \& Environment, 123, 247-260, 2008.

Arshad, M., Silvestre, J., Pinelli, E., Kallerhoff, J., Kaemmerer, M., Tarigo, A., Shahid, M., Guiresse, M., Pradere, P., and Dumat, C.: A field study of lead phytoextraction by various scented Pelargonium cultivars, Chemosphere, 71, 2187-2192, 2008.

ATSDR, U.: United States Agency for Toxic Substances and Disease Registry.Toxicological Profile for lead, U.S. Department of Health and Human Services, 2007. 1-582, 2007.

Banuelos, G. S.: Phyto-products may be essential for sustainability and implementation of phytoremediation, Environmental pollution (Barking, Essex : 1987), 144, 19-23, 2006.

Bech, J., Duran, P., Roca, N., Poma, W., Sánchez, I., Barceló, J., Boluda, R., Roca-Pérez, L., and Poschenrieder, C.: Shoot accumulation of several trace elements in native plant species from contaminated soils in the Peruvian Andes, Journal of Geochemical Exploration, 113, 106-111, 2012a.

Bech, J., Duran, P., Roca, N., Poma, W., Sánchez, I., Roca-Pérez, L., Boluda, R., Barceló, J., and Poschenrieder, $\mathrm{C}$.: Accumulation of $\mathrm{Pb}$ and $\mathrm{Zn}$ in Bidens triplinervia and Senecio sp. spontaneous species from mine spoils in Peru and their potential use in phytoremediation, Journal of Geochemical Exploration, 123, 109-113, 2012b.

Bhargava, A., Carmona, F. F., Bhargava, M., and Srivastava, S.: Approaches for enhanced phytoextraction of heavy metals, Journal of Environmental Management, 105, 103-120, 2012. Bharti, S. and Kumar Banerjee, T.: Phytoremediation of the coalmine effluent, Ecotoxicology and Environmental Safety, 81, 36-42, 2012.

Bolan, N., Kunhikrishnan, A., Thangarajan, R., Kumpiene, J., Park, J., Makino, T., Kirkham, M. B., and Scheckel, K.: Remediation of heavy metal(loid)s contaminated soils - To mobilize or to immobilize?, Journal of Hazardous Materials, 266, 141-166, 2014.

Boominathan, R., Saha-Chaudhury, N. M., Sahajwalla, V., and Doran, P. M.: Production of nickel bioore from hyperaccumulator plant biomass: applications in phytomining, Biotechnology and bioengineering, 86, 243-250, 2004.

Brooks, R. R.: Copper and cobalt uptake by Haumaniastrum species, Plant and Soil, 48, 541-544, 1977.

Brooks, R. R. and Robinson, B. H.: The potential use of hyperaccumulators and other plants in phytomining. In: Brooks RR. (Ed.), Plants that Hyperaccumulate Heavy Metals: Their Role in Phytoremediation, Microbiology, Archaeology, Mineral Exploration and Phytomining, CAB International, Wallingford, UK. 327-356., 1998. 1998. 
Solid Earth Discuss., https://doi.org/10.5194/se-2017-75

Manuscript under review for journal Solid Earth

Discussion started: 6 November 2017

(c) Author(s) 2017. CC BY 4.0 License.

Bu, J., Sun, Z., Zhou, A., Xu, Y., Ma, R., Wei, W., and Liu, M.: Heavy Metals in Surface Soils in the Upper Reaches of the Heihe River, Northeastern Tibetan Plateau, China, International Journal of Environmental Research and Public Health, 13, 247, 2016.

Cannon: Geochemistry of rocks and related soils and vegetation in the Yellow Cat area, Grand County, Utah, 1964. 1964.

Cao, A., Carucci, A., Lai, T., La Colla, P., and Tamburini, E.: Effect of biodegradable chelating agents on heavy metals phytoextraction with Mirabilis jalapa and on its associated bacteria, European Journal of Soil Biology, 43, 200-206, 2007.

Chaney, R. L., Angle, J. S., Baker, A. J. M., and Li, J. M.: Method for phytomining of nickel, cobalt, and other metal from soil, US Patent \# 5: , 1998. 711-784, 1998.

Chehregani, A., Noori, M., and Yazdi, H. L.: Phytoremediation of heavy-metal-polluted soils:

Screening for new accumulator plants in Angouran mine (Iran) and evaluation of removal ability, Ecotoxicology and Environmental Safety, 72, 1349-1353, 2009.

Chen, B.-C., Lai, H.-Y., and Juang, K.-W.: Model evaluation of plant metal content and biomass yield for the phytoextraction of heavy metals by switchgrass, Ecotoxicology and Environmental Safety, 80, 393-400, 2012.

Chen, L., Luo, S., Li, X., Wan, Y., Chen, J., and Liu, C.: Interaction of Cd-hyperaccumulator Solanum nigrum L. and functional endophyte Pseudomonas sp. Lk9 on soil heavy metals uptake, Soil Biology and Biochemistry, 68, 300-308, 2014.

Chen, X., Xia, X., Zhao, Y., and Zhang, P.: Heavy metal concentrations in roadside soils and correlation with urban traffic in Beijing, China, Journal of Hazardous Materials, 181, 640-646, 2010. Chen, Y., Li, X., and Shen, Z.: Leaching and uptake of heavy metals by ten different species of plants during an EDTA-assisted phytoextraction process, Chemosphere, 57, 187-196, 2004.

Chen, Y. X., Lin, Q., Luo, Y. M., He, Y. F., Zhen, S. J., Yu, Y. L., Tian, G. M., and Wong, M. H.: The role of citric acid on the phytoremediation of heavy metal contaminated soil, Chemosphere, 50, 807-811, 2003.

Cho, Y., Bolick, J. A., and Butcher, D. J.: Phytoremediation of lead with green onions (Allium fistulosum) and uptake of arsenic compounds by moonlight ferns (Pteris cretica cv Mayii), Microchemical Journal, 91, 6-8, 2009.

Conesa, H. M., Evangelou, M. W. H., Robinson, B. H., and Schulin, R.: A Critical View of Current State of Phytotechnologies to Remediate Soils: Still a Promising Tool?, The Scientific World Journal, 2012, 173829, 2012.

Daud, M. K., Ali, S., Variath, M. T., and Zhu, S. J.: Differential physiological, ultramorphological and metabolic responses of cotton cultivars under cadmium stress, Chemosphere, 93, 2593-2602, 2013. Dechamps, C., Roosens, N. H., Hotte, C., and Meerts, P.: Growth and mineral element composition in two ecotypes of Thlaspi caerulescens on Cd contaminated soil, Plant and Soil, 273, 327-335, 2005.

Deng, G., Li, M., Li, H., Yin, L., and Li, W.: Exposure to cadmium causes declines in growth and photosynthesis in the endangered aquatic fern (Ceratopteris pteridoides), Aquatic Botany, 112, 2332, 2014

Dermont, G., Bergeron, M., Mercier, G., and Richer-Laflèche, M.: Metal-Contaminated Soils: Remediation Practices and Treatment Technologies, Practice Periodical of Hazardous Toxic \& Radioactive Waste Management, 12, 188-209, 2008.

Epelde, L., Hernández-Allica, J., Becerril, J. M., Blanco, F., and Garbisu, C.: Effects of chelates on plants and soil microbial community: Comparison of EDTA and EDDS for lead phytoextraction, Science of The Total Environment, 401, 21-28, 2008. 
Solid Earth Discuss., https://doi.org/10.5194/se-2017-75

Manuscript under review for journal Solid Earth

Discussion started: 6 November 2017

(c) Author(s) 2017. CC BY 4.0 License.

Epelde, L., Mijangos, I., Becerril, J. M., and Garbisu, C.: Soil microbial community as bioindicator of
the recovery of soil functioning derived from metal phytoextraction with sorghum, Soil Biology and Biochemistry, 41, 1788-1794, 2009.

Erdei, L., Mezôsi, G., Mécs, I., Vass, I., Fôglein, F., and Bulik, L.: Phytoremediation as a program for decontamination of heavy-metal polluted environment, Acta Biologica Szegediensis, 2005. 75-76, 2005.

Escarre, J., LefÈBvre, C., Gruber, W., Leblanc, M., Lepart, J., RivièRe, Y., and Delay, B.: Zinc and cadmium hyperaccumulation by Thlaspi caerulescens from metalliferous and nonmetalliferous sites in the Mediterranean area: implications for phytoremediation, New Phytologist, 145, 429-437, 2000.

Evangelou, M. W., Daghan, H., and Schaeffer, A.: The influence of humic acids on the phytoextraction of cadmium from soil, Chemosphere, 57, 207-213, 2004.

Evangelou, M. W. H. and Deram, A.: Phytomanagement: A realistic approach to soil remediating phytotechnologies with new challenges for plant science, 2, 2014.

Evangelou, M. W. H., Ebel, M., Koerner, A., and Schaeffer, A.: Hydrolysed wool: A novel chelating agent for metal chelant-assisted phytoextraction from soil, Chemosphere, 72, 525-531, 2008.

Evangelou, M. W. H., Ebel, M., and Schaeffer, A.: Evaluation of the effect of small organic acids on phytoextraction of $\mathrm{Cu}$ and $\mathrm{Pb}$ from soil with tobacco Nicotiana tabacum, Chemosphere, 63, 9961004, 2006.

Freitas, E. V., Nascimento, C. W., Souza, A., and Silva, F. B.: Citric acid-assisted phytoextraction of lead: A field experiment, Chemosphere, 92, 213-217, 2013.

Gabos, M. B., Abreu, C. A. d., and Coscione, A. R.: EDTA assisted phytorremediation of a Pb contamined soil: metal leaching and uptake by jack beans, Scientia Agricola, 66, 506-514, 2009. Gallego, S. M., Pena, L. B., Barcia, R. A., Azpilicueta, C. E., lannone, M. F., Rosales, E. P., Zawoznik, M. S., Groppa, M. D., and Benavides, M. P.: Unravelling cadmium toxicity and tolerance in plants: Insight into regulatory mechanisms, Environmental and Experimental Botany, 83, 33-46, 2012. Gao, Y., Miao, C., Xia, J., Luo, C., Mao, L., Zhou, P., and Shi, W.: Effect of citric acid on phytoextraction and antioxidative defense in Solanum nigrum L. as a hyperaccumulator under $\mathrm{Cd}$ and $\mathrm{Pb}$ combined pollution, Environmental Earth Sciences, 65, 1923-1932, 2012.

García, G., Faz, Á., and Cunha, M.: Performance of Piptatherum miliaceum (Smilo grass) in edaphic $\mathrm{Pb}$ and $\mathrm{Zn}$ phytoremediation over a short growth period, International Biodeterioration \& Biodegradation, 54, 245-250, 2004.

Gopal, R. and Rizvi, A. H.: Excess lead alters growth, metabolism and translocation of certain nutrients in radish, Chemosphere, 70, 1539-1544, 2008.

Gupta, D. K., Huang, H. G., Yang, X. E., Razafindrabe, B. H., and Inouhe, M.: The detoxification of lead in Sedum alfredii $\mathrm{H}$. is not related to phytochelatins but the glutathione, J Hazard Mater, 177, 437-444, 2010.

Ha, N. T., Sakakibara, M., and Sano, S.: Accumulation of Indium and other heavy metals by Eleocharis acicularis: An option for phytoremediation and phytomining, Bioresour Technol, 102, 2228-2234, 2011.

Hadi, F., Bano, A., and Fuller, M. P.: The improved phytoextraction of lead (Pb) and the growth of maize (Zeamays L.): the role of plant growth regulators (GA3 and IAA) and EDTA alone and in combinations, Chemosphere, 80, 457-462, 2010.

Harris, A. T., Naidoo, K., Nokes, J., Walker, T., and Orton, F.: Indicative assessment of the feasibility of Ni and Au phytomining in Australia, Journal of Cleaner Production, 17, 194-200, 2009. 
Solid Earth Discuss., https://doi.org/10.5194/se-2017-75

Manuscript under review for journal Solid Earth

Discussion started: 6 November 2017

(c) Author(s) 2017. CC BY 4.0 License.

He, L. Y., Chen, Z. J., Ren, G. D., Zhang, Y. F., Qian, M., and Sheng, X. F.: Increased cadmium and lead uptake of a cadmium hyperaccumulator tomato by cadmium-resistant bacteria, Ecotoxicol Environ Saf, 72, 1343-1348, 2009.

Hseu, Z.-Y., Jien, S.-H., Wang, S.-H., and Deng, H.-W.: Using EDDS and NTA for enhanced phytoextraction of $\mathrm{Cd}$ by water spinach, Journal of Environmental Management, 117, 58-64, 2013. Huang, J. W. and Cunningham, S. D.: Lead phytoextraction: species variation in lead uptake and translocation, New Phytologist, 134, 75-84, 1996.

Jaffré, T.: Etude écologique du peuplement végétal des sols dérivés de roches ultrabasiques en Nouvelle Calédonie, 1980. 1980.

Jiang, Y., Lei, M., Duan, L., and Longhurst, P.: Integrating phytoremediation with biomass valorisation and critical element recovery: A UK contaminated land perspective, Biomass and Bioenergy, 83, 328-339, 2015.

Kaimi, E., Mukaidani, T., Miyoshi, S., and Tamaki, M.: Ryegrass enhancement of biodegradation in diesel-contaminated soil, Environmental \& Experimental Botany, 55, 110-119, 2006.

Kirkham, M. B.: Cadmium in plants on polluted soils: Effects of soil factors, hyperaccumulation, and amendments, Geoderma, 137, 19-32, 2006.

Leblanc, M., Petit, D., Deram, A., Robinson, B. H., Brooks, R. R., Leblanc, M., Petit, D., and Brooks, R. R.: The phytomining and environmental significance of hyperaccumulation of thallium by lberis intermedia from Southern France, Economic Geology, 94, 109-113, 1999.

Lehmann, J.: Bio-energy in the black, Frontiers in Ecology \& the Environment, 5, 381-387, 2007. Li, C., Jiang, W., Ma, N., Zhu, Y., Dong, X., Wang, D., Meng, X., and Xu, Y.: Bioaccumulation of cadmium by growing Zygosaccharomyces rouxii and Saccharomyces cerevisiae, Bioresource Technology, 155, 116-121, 2014.

Li, Y., Chaney, R., Brewer, E., Roseberg, R., Angle, J. S., Baker, A., Reeves, R., and Nelkin, J.: Development of a technology for commercial phytoextraction of nickel: economic and technical considerations, Plant and Soil, 249, 107-115, 2003.

Liang, J., Shohag, M. J., Yang, X., Tian, S., Zhang, Y., Feng, Y., and He, Z.: Role of sulfur assimilation pathway in cadmium hyperaccumulation by Sedum alfredii Hance, Ecotoxicology \& Environmental Safety, 100, 159-165, 2014.

Liu, X., Peng, K., Wang, A., Lian, C., and Shen, Z.: Cadmium accumulation and distribution in populations of Phytolacca americana L. and the role of transpiration, Chemosphere, 78, 1136-1141, 2010.

Liu, Z., He, X., Wei, C., Yuan, F., Yan, K., and Tao, D.: Accumulation and tolerance characteristics of cadmium in a potential hyperaccumulator - Lonicera japonica Thunb, Journal of Hazardous Materials, 169, 170-175, 2009.

Lomonte, C., Sgherri, C., Baker, A. J. M., Kolev, S. D., and Navari-Izzo, F.: Antioxidative response of Atriplex codonocarpa to mercury, Environmental \& Experimental Botany, 69, 9-16, 2010.

Luo, C., Shen, Z., and Li, X.: Enhanced phytoextraction of $\mathrm{Cu}, \mathrm{Pb}, \mathrm{Zn}$ and $\mathrm{Cd}$ with EDTA and EDDS, Chemosphere, 59, 1-11, 2005.

Mahar, A., Wang, P., Ali, A., Awasthi, M. K., Lahori, A. H., Wang, Q., Li, R., and Zhang, Z.: Challenges and opportunities in the phytoremediation of heavy metals contaminated soils: A review, Ecotoxicology \& Environmental Safety, 126, 111-121, 2016.

Marmiroli, M., Imperiale, D., Maestri, E., and Marmiroli, N.: The response of Populus spp. to cadmium stress: chemical, morphological and proteomics study, Chemosphere, 93, 1333-1344, 2013. 
Solid Earth Discuss., https://doi.org/10.5194/se-2017-75

Manuscript under review for journal Solid Earth

Discussion started: 6 November 2017

(c) Author(s) 2017. CC BY 4.0 License.

Mcgrath, S. P. and Zhao, F. J.: Phytoextraction of metals and metalloids from contaminated soils, Current Opinion in Biotechnology, 14, 277-282, 2003.

Meers, E., Vandecasteele, B., Ruttens, A., Vangronsveld, J., and Tack, F. M. G.: Potential of five willow species (Salix spp.) for phytoextraction of heavy metals, Environmental \& Experimental Botany, 60, 57-68, 2007. Meighan, M. M., Fenus, T., Karey, E., and Macneil, J.: The impact of EDTA on the rate of accumulation and root/shoot partitioning of cadmium in mature dwarf sunflowers, Chemosphere, 83, 1539, 2011.

Morais, I., Campos, J. S., Favas, P. J. C., Pratas, J., Pita, F., and Prasad, M. N. V.: Nickel accumulation by Alyssum serpyllifolium subsp. lusitanicum (Brassicaceae) from serpentine soils of Bragança and Morais (Portugal) ultramafic massifs: plant-soil relationships and prospects for phytomining, Australian Journal of Botany, 63, 17-30, 2015.

Msuya, F. A., Brooks, R. R., and Anderson, C. W. N.: Chemically-induced uptake of gold by root crops: Its significance for phytomining, Gold Bulletin, 33, 134-137, 2000.

Nagajyoti, P. C., Lee, K. D., and Sreekanth, T. V. M.: Heavy metals, occurrence and toxicity for plants: a review, Environmental Chemistry Letters, 8, 199-216, 2010.

Nyquist, J. and Greger, M.: Response of two wetland plant species to $\mathrm{Cd}$ exposure at low and neutral pH, Environmental \& Experimental Botany, 65, 417-424, 2009.

Pacyna, J. M., Pacyna, E. G., and Aas, W.: Changes Of Emissions And Atmospheric Deposition Of Mercury,lead,and Cadmium, Atmospheric Environment, 43, 117-127, 2009.

Padmaja, K., Prasad, D. D. K., and Prasad, A. R. K.: Inhibition of chlorophyll synthesis in Phaseolus vulgaris L. seedlings by cadmium acetate, Photosynthetica, 1990. 1990.

Pan, Y. P. and Wang, Y. S.: Atmospheric wet and dry deposition of trace elements at 10 sites in Northern China, Atmospheric Chemistry \& Physics, 14, 20647-20676, 2014.

Peng, K., Luo, C., You, W., Lian, C., Li, X., and Shen, Z.: Manganese uptake and interactions with cadmium in the hyperaccumulator--Phytolacca Americana L, Journal of Hazardous Materials, 154, 674-681, 2008.

Perronnet, K., Schwartz, C., and Morel, J. L.: Distribution of cadmium and zinc in the hyperaccumulator Thlaspi caerulescens grown on multicontaminated soil, Plant and Soil, 249, 1925, 2003

Pilonsmits, E.: Annual review of plant biology, Annual Review of Plant Biology, 56, 2005.

Prasad, M. N. V.: Phytoremediation of Metal-Polluted Ecosystems: Hype for Commercialization, Russian Journal of Plant Physiology, 50, 686-701, 2003.

Pulford, I. D. and Watson, C.: Phytoremediation of heavy metal-contaminated land by trees-a review, Environment International, 29, 529, 2003.

Qaim, M., Stein, A. J., and Meenakshi, J. V.: Economics of biofortification, Agricultural Economics, 37, 119-133, 2007.

Qiu, R. L., Zhao, X., Tang, Y. T., Yu, F. M., and Hu, P. J.: Antioxidative response to Cd in a newly discovered cadmium hyperaccumulator, Arabis paniculata F, Chemosphere, 74, 6-12, 2009. Rascio, N. and Navariizzo, F.: Heavy metal hyperaccumulating plants: how and why do they do it? And what makes them so interesting?, Plant Science An International Journal of Experimental Plant Biology, 180, 169, 2011.

Reeves, R. D., Baker, A. J. M., and Brooks, R. R.: Abnormal accumulation of trace metals by plants, 1996. 1996.

Reeves, R. D. and Brooks, R. R.: Hyperaccumulation of lead and zinc by two metallophytes from mining areas of Central Europe, Environmental Pollution, 31, 277-285, 1983. 
Solid Earth Discuss., https://doi.org/10.5194/se-2017-75

Manuscript under review for journal Solid Earth

Discussion started: 6 November 2017

(c) Author(s) 2017. CC BY 4.0 License.

Reeves, R. D., Schwartz, C., Morel, J. L., and Edmondson, J.: Distribution and Metal-Accumulating Behavior of Thlaspi caerulescens and Associated Metallophytes in France, International journal of phytoremediation, 3, 145-172., 2001.

Robinson, B. H., Brooks, R. R., Howes, A. W., Kirkman, J. H., and Gregg, P. E. H.: The potential of the high-biomass nickel hyperaccumulator Berkheya coddii for phytoremediation and phytomining, Journal of Geochemical Exploration, 60, 115-126, 1997.

Robinson, B. H. and Mcgrath, S. P.: Uptake and distribution of nickel and other metals in the hyperaccumulator Berkheya coddii, New Phytologist, 158, 279-285, 2003.

Rosenfeld, P. E. and Henry, C. L.: Activated carbon and wood ash sorption of wastewater, compost, and biosolids odorants, Water Environment Research, 73, 388-393, 2001.

Saifullah, Zia, M. H., Meers, E., Ghafoor, A., Murtaza, G., Sabir, M., Zia-Ur-Rehman, M., and Tack, F. M.: Chemically enhanced phytoextraction of $\mathrm{Pb}$ by wheat in texturally different soils, Chemosphere, 79, 652-658, 2010.

Salazar, M. J. and Pignata, M. L.: Lead accumulation in plants grown in polluted soils. Screening of native species for phytoremediation, Journal of Geochemical Exploration, 137, 29-36, 2014.

Salazar, M. J. and Pignata, M. L.: Lead accumulation in plants grown in polluted soils. Screening of native species for phytoremediation, Journal of Geochemical Exploration, 137, 29-36, 2013.

Schor-Fumbarov, T., Keilin, Z., and Tel-Or, E.: Characterization of cadmium uptake by the water lily Nymphaea aurora, International journal of phytoremediation, 5, 169-179, 2003.

Sekhar, K. C., Kamala, C. T., Chary, N. S., Balaram, V., and Garcia, G.: Potential of Hemidesmus indicus for phytoextraction of lead from industrially contaminated soils, Chemosphere, 58, 507-514, 2005.

Sheoran, V., Sheoran, A. S., and Poonia, P.: Phytomining of gold: A review, Journal of Geochemical Exploration, 128, 42-50, 2013.

Sheoran, V., Sheoran, A. S., and Poonia, P.: Phytomining: A review, Minerals Engineering, 22, 10071019, 2009.

Singh, R., Tripathi, R. D., Dwivedi, S., Kumar, A., Trivedi, P. K., and Chakrabarty, D.: Lead bioaccumulation potential of an aquatic macrophyte Najas indica are related to antioxidant system, Bioresource Technology, 101, 3025-3032, 2010.

Smolińska, B. and K, C.: EDTA and urease effects on $\mathrm{Hg}$ accumulation by Lepidium sativum, Chemosphere, 69, 1388-1395, 2007.

Song, X., Hu, X., Ji, P., Li, Y., Chi, G., and Song, Y.: Phytoremediation of cadmium-contaminated farmland soil by the hyperaccumulator Beta vulgaris L. var. cicla, Bulletin of Environmental Contamination and Toxicology, 88, 623-626, 2012.

Sun, Y., Zhou, Q., Lin, W., and Liu, W.: Cadmium tolerance and accumulation characteristics of Bidens pilosa L. as a potential Cd-hyperaccumulator, Journal of Hazardous Materials, 161, 808-814, 2009a.

Sun, Y. B., Zhou, Q. X., An, J., Liu, W. T., and Liu, R.: Chelator-Enhanced Phytoextraction of Heavy Metals from Contaminated Soil Irrigated by Industrial Wastewater with the Hyperaccumulator Plant (Sedum alfredii Hance), Geoderma, 150, 106-112, 2009b.

Tang, Y. T., Qiu, R. L., Zeng, X. W., Ying, R. R., Yu, F. M., and Zhou, X. Y.: Lead, zinc, cadmium hyperaccumulation and growth stimulation in Arabis paniculata Franch, Environmental \& Experimental Botany, 66, 126-134, 2009.

Tangahu, B. V., Abdullah, S. R. S., Basri, H., Idris, M., Anuar, N., and Mukhlisin, M.: A Review on Heavy Metals (As, $\mathrm{Pb}$, and $\mathrm{Hg}$ ) Uptake by Plants through Phytoremediation, International Journal of Chemical Engineering, 2011, 2011. 
Solid Earth Discuss., https://doi.org/10.5194/se-2017-75

Manuscript under review for journal Solid Earth

Discussion started: 6 November 2017

(c) Author(s) 2017. CC BY 4.0 License.

Thangavel, P. and Subbhuraam, C. V.: Phytoextraction: Role of hyperaccumulators in metal contaminated soils, Proceedings Indian Natural Science Academy. Part B, 70, 109-130, 2004. Vollenweider, P., Cosio, C., Günthardt-Goerg, M. S., and Keller, C.: Localization and effects of cadmium in leaves of a cadmium-tolerant willow (Salix viminalis L.) Part II Microlocalization and cellular effects of cadmium. , Environmental \& Experimental Botany, 58, 64-74, 2006.

Wadge, A. and Hutton, M.: The cadmium and lead content of suspended particulate matter emitted from a U.K. refuse incinerator, The Science of the total environment, 67, 91-95, 1987. Wahid, A., Ghani, A., and Javed, F.: Effect of cadmium on photosynthesis, nutrition and growth of mungbean, Agronomy for Sustainable Development, 28, 273-280, 2008.

Walraven, N., van Os, B. J., Klaver, G. T., Middelburg, J. J., and Davies, G. R.: The lead (Pb) isotope signature, behaviour and fate of traffic-related lead pollution in roadside soils in The Netherlands, Science of the Total Environment, 472, 888-900, 2014.

Wang, H.-q., LUSi-jin, LIhua, and YAOZhi-hua: EDTA-enhanced phytoremediation of lead contaminated soil by Bidens maximowicziana, Journal of Environmental Sciences, 19, 1496-1499, 2007.

Wang, K. S., Huang, L. C., Lee, H. S., Chen, P. Y., and Chang, S. H.: Phytoextraction of cadmium by Ipomoea aquatica (water spinach) in hydroponic solution: effects of cadmium speciation, Chemosphere, 72, 666-672, 2008.

Wei, J. L., Lai, H. Y., and Chen, Z. S.: Chelator effects on bioconcentration and translocation of cadmium by hyperaccumulators, Tagetes patula and Impatiens walleriana, Ecotoxicology \& Environmental Safety, 84, 173-178, 2012.

Wei, S. H. and Zhou, Q. X.: Phytoremediation of cadmium-contaminated soils by Rorippa globosa using two-phase planting, Environmental Science and Pollution Research, 13, 151-155, 2006. Wenhao, Y., Hong, H., Mei, R., and Wuzhong, N.: Changes of microbial properties in (near-) rhizosphere soils after phytoextraction by Sedum alfredii $\mathrm{H}$ : A rhizobox approach with an artificial Cd-contaminated soil, Applied Soil Ecology, 72, 14-21, 2013.

Wenzel, W. W. and Jockwer, F.: Accumulation of heavy metals in plants grown on mineralised soils of the Austrian Alps, Environmental Pollution, 104, 145-155, 1999.

WHO: (World Health Organization) Safety evaluation of certain food additives and contaminants, WHO Food Additives, Series No. 44., 2000.

Wilson-Corral, V., Anderson, C., Rodriguez-Lopez, M., Arenas-Vargas, M., and Lopez-Perez, J.: Phytoextraction of gold and copper from mine tailings with Helianthus annuus L. and Kalanchoe serrata L, Minerals Engineering, 24, 1488-1494, 2011.

Xiao, X., Chen, T., An, Z., Lei, M., Huang, Z., Liao, X., and Liu, Y.: Potential of Pteris vittata L. for phytoremediation of sites co-contaminated with cadmium and arsenic: the tolerance and accumulation, 环境科学学报(英文版), 20, 62-67, 2008.

Xie, W. Y., Huang, Q., Li, G., Rensing, C., and Zhu, Y. G.: Cadmium accumulation in the rootless macrophyte Wolffia globosa and its potential for phytoremediation, International Journal of Phytoremediation, 2013.

Xiong, Z. T.: Bioaccumulation and physiological effects of excess lead in a roadside pioneer species Sonchus oleraceus L, Environmental Pollution, 97, 275, 1997.

Xu, Q., Min, H., Cai, S., Fu, Y., Sha, S., Xie, K., and Du, K.: Subcellular distribution and toxicity of cadmium in Potamogeton crispus L, Chemosphere, 89, 114-120, 2012.

Yang, X. E. and Stoffella, P. J.: Cadmium tolerance and hyperaccumulation in a new Znhyperaccumulating plant species (Sedum alfredii Hance), Plant and Soil, 259, 181-189, 2004. 
Solid Earth Discuss., https://doi.org/10.5194/se-2017-75

Manuscript under review for journal Solid Earth

Discussion started: 6 November 2017

(c) Author(s) 2017. CC BY 4.0 License.

Zaier, H., Ghnaya, T., Lakhdar, A., Baioui, R., Ghabriche, R., Mnasri, M., Sghair, S., Lutts, S., and Abdelly, C.: Comparative study of $\mathrm{Pb}$-phytoextraction potential in Sesuvium portulacastrum and Brassica juncea: tolerance and accumulation, Journal of hazardous materials, 183, 609-615, $2010 \mathrm{a}$. Zaier, H., Ghnaya, T., Lakhdar, A., Baioui, R., Ghabriche, R., Mnasri, M., Sghair, S., Lutts, S., and Abdelly, C.: Comparative study of $\mathrm{Pb}$-phytoextraction potential in Sesuvium portulacastrum and Brassica juncea: tolerance and accumulation, Journal of Hazardous Materials, 183, 609-615, $2010 \mathrm{~b}$. Zhang, W. L., Du, Y., Zhai, M. M., and Shang, Q.: Cadmium exposure and its health effects: a 19-year follow-up study of a polluted area in China, Science of the Total Environment, s 470-471, 224-228, 2014.

Zhang, X., Lin, L., Chen, M., Zhu, Z., Yang, W., Chen, B., Yang, X., and An, Q.: A nonpathogenic Fusarium oxysporum strain enhances phytoextraction of heavy metals by the hyperaccumulator Sedum alfredii Hance, Journal of Hazardous Materials, s 229-230, 361-370, 2012.

Zhao, F. J. and Mcgrath, S. P.: Biofortification and phytoremediation, Current Opinion in Plant Biology, 12, 373-380, 2009.

Zhao, K., Liu, X. M., Xu, J. M., and Selim, H. M.: Heavy metal contaminations in a soil-rice system: identification of spatial dependence in relation to soil properties of paddy fields, Journal of Hazardous Materials, 181, 778-787, 2010.

Zhou, H., Zhou, X., Zeng, M., Liao, B. H., Liu, L., Yang, W. T., Wu, Y. M., Qiu, Q. Y., and Wang, Y. J.: Effects of combined amendments on heavy metal accumulation in rice (Oryza sativa L.) planted on contaminated paddy soil, Ecotoxicology \& Environmental Safety, 101, 226-232, 2014. 